Kragujevac Journal of Mathematics

Volume 44(2) (2020), Pages 217-236.

\title{
SOFT INTERIOR-HYPERIDEALS IN LEFT REGULAR LA-SEMIHYPERGROUPS
}

\author{
M. Y. ABBASI ${ }^{1}$, S. A. KHAN ${ }^{1}$, A. F. TALEE ${ }^{1}$, AND A. KHAN ${ }^{2}$ \\ ABSTRACT. This paper is a contribution to the study of the effective content of LA- \\ hyperstructure. In this paper, we introduce the notion of soft interior-hyperideals. \\ Further, we give several basic properties of these notions and provide different \\ important characterizations in terms of soft interior hyperideals.
}

\section{INTRODUCTION}

Marty [23] introduced the notion of algebraic hyperstructures as natural generalization of classical algebraic structures. The difference between classical algebraic structures and algebraic hyperstructures is that, in algebraic structures the composition of two elements is an element while in algebraic hyperstructure the composition of two elements is a non-empty set. Koskas introduced the notion of semihypergroups. Hasankhani [15] defined ideals in right (left) semihypergroups and discussed some hyper versions of Green's relations.

The concept of LA-semigroup was given by Kazim and Naseeruddin [17]. Faisal et al. [34] characterized left regular LA-semigroup in terms of fuzzy interior ideals. Hila and Dine [16] defined LA-semihypergroups. They studied several properties of hyperideals of LA-semihypergroup. Yaqoob et al. [30] gave some characterizations of LA-semihypergroups using left, right and interior-hyperideals. Yousafzai and Corsini [33] extended the theory of an LA-semigroup in terms of their one sided ideals. They characterized the class of an intra-regular LA-semihypergroup using one-sided hyperideals. Amjad [2] defined generalized hyperideals in locally associative left almost semihypergroups and in [3] they studied pure LA-semihypergroups. Yaqoob and

Key words and phrases. LA-semihypergroups, soft interior-hyperideals, left regular LAsemihypergroups.

2010 Mathematics Subject Classification. Primary: 20N20. Secondary: 20M99, 03 E75.

DOI 10.46793/KgJMat2002.217A

Received: December 22, 2017.

Accepted: March 30, 2018. 
Gulistan [31] studied hyperideals and $M$-hypersystem in partially ordered left almost semihypergroups. Recently, many authors [5, 6, 13, 14, 18-20, 28, 29, 32] have worked on LA-semihypergroups.

Our world is surrounded by uncertainties and ambiguities. We pass through many uncertainties in our daily life. Therefore, it is necessary to prepare a model so that we deal such uncertainties and ambiguities. Initially, probability theory was the only mathematical concept for dealing some unplanned activities. To handle some special kind of activity known as fuzziness, Zadeh [35] introduced the notion of fuzzy set as an extension of classical set theory. But there was a difficulty for membership function. How to set the membership function in each particular case. We cannot impose only one way to set the membership function. The reason for these difficulties is, possibly, the inadequacy of the parametrization tool of the theory. To remove this difficulty, Molodtsov [24] introduced a mathematical tool for dealing with hesitant, fuzzy, unpredictable and unsure articles known as soft set. A soft set is a collection of approximate descriptions of an object. Each approximate description has two parts: a predicate and an approximate value set. Further, Maji et al. [22] defined many applications in soft sets. After the beginning of soft set theory, many authors gave a new view to classical mathematics. Cagman and Aktas proposed the concept of soft algebraic structure. They introduced soft group theory [1] and gave the definition of soft group which is analogous to the rough group definition. They correlate soft sets with rough sets and fuzzy sets. After that many authors $[8,12,27]$ have worked on soft algebraic structures. Cagman et al. [7] gave a new approach to soft group definition called soft intersection group. This approach is depends on the insertion and intersection of sets. Anvariyeh et al. [4] initiated soft semihypergroups by using the soft set theory. Sezgin [26] studied soft set theory in LA-semigroup with the concept of soft intersection LA-semigroups and soft intersection LA-ideals. Naz and Shabir [25] investigated the basic terms and properties of soft sets. They relate soft sets with the concept of semihypergroups. Farooq et al. [11] characterized regular and left regular ordered semihypergroups using intersection soft generalized bi-hyperideals. Khan et al. [21] introduced the notion of soft intersection (S.I.) hyperideals in LA-semihypergroups and gave some characterizations.

In this paper, we introduce soft interior-hyperideals through new approach called soft intersection (briefly, S.I.) and establish some of their elementary properties. We also define the concept of soft semiprime and study some results on them. We characterize left regular LA-semihypergroups in terms of soft interior-hyperideals and prove that in a left regular LA-semihypergroup, soft interior-hyperideals and soft bi-hyperideals coincide.

\section{Preliminaries}

Throughout this paper we represent:

H : LA-semihypergroup, $\mathcal{U}$ : an initial universe, 
$E$ : a set of parameters,

$\mathbf{H}(\mathcal{U})$ : set of all soft sets of $\mathbf{H}$ over $\mathcal{U}$, $P(\mathcal{U})$ : the powerset of $\mathcal{U}$.

Definition 2.1 ([9, 10]). Let $\mathbf{H}$ be a non-empty set and let $\wp^{*}(\mathbf{H})$ be the set of all non-empty subsets of $\mathbf{H}$. A hyperoperation on $\mathbf{H}$ is a map $\underline{\mathrm{o}}: \mathbf{H} \times \mathbf{H} \rightarrow \wp^{*}(\mathbf{H})$ and $(\mathbf{H}, \underline{\text { o }})$ is called a hypergroupoid.

Definition $2.2([9,10])$. A hypergroupoid $(\mathbf{H}, \underline{\mathrm{o}})$ is called a semihypergroup if for all $x, y, z$ of $\mathbf{H}$ we have $(x \underline{\mathrm{o}} y) \underline{\mathrm{o}} z=x \underline{\mathrm{o}}(y \underline{\mathrm{o}} z)$, which means that

$$
\bigcup_{u \in x \underline{\mathrm{O}} y} u \underline{\mathrm{O}} z=\bigcup_{v \in y \underline{\mathrm{O}} z} x \underline{\mathrm{O}} v .
$$

If $x \in \mathbf{H}$ and $A, B$ are non-empty subsets of $\mathbf{H}$, then we denote

$$
A \underline{\mathrm{o}} B=\bigcup_{a \in A, b \in B} a \underline{\mathrm{o}} b, x \circ A=\{x\} \underline{\mathrm{o}} A \text { and } A \underline{\mathrm{o}} x=A \underline{\mathrm{o}}\{x\} \text {. }
$$

Definition 2.3 ([16]). Let $\mathbf{H}$ be non-empty set. A hypergroupoid $\mathbf{H}$ is called an LA-semihypergroup if for every $x, y, z \in \mathbf{H}$, we have

$$
(x \underline{\mathrm{o}} y) \underline{\mathrm{o}} z=(z \circ y) \underline{\mathrm{o}} x .
$$

The law is called left invertive law. Every LA-semihypergroup satisfies the following law:

$$
(x \underline{\mathrm{o}} y) \underline{\mathrm{o}}(z \underline{\mathrm{o}} w)=(x \underline{\mathrm{o}} z) \underline{\mathrm{o}}(y \underline{\mathrm{o}} w),
$$

for all $w, x, y, z \in H$. This law is known as medial law.

Definition 2.4 ([30]). Let $\mathbf{H}$ be an LA-semihypergroup, then an element $e \in \mathbf{H}$ is called left identity (resp., pure left identity) if for all $a \in \mathbf{H}, a \in e \underline{\text { o }} a$ (resp., $a=e \underline{\mathrm{o}} a)$.

An LA- semihypergroup $(\mathbf{H}, \underline{o})$ with pure left identity satisfy the following law for all $w, x, y, z \in \mathbf{H}$ :

$$
(x \underline{\mathrm{o}} y) \underline{\mathrm{o}}(z \underline{\mathrm{o}} w)=(w \underline{\mathrm{o}} z) \underline{\mathrm{o}}(y \underline{\mathrm{o}} x),
$$

called a paramedial law, and

$$
x \underline{\mathrm{o}}(y \underline{\mathrm{o}} z)=y \underline{\mathrm{o}}(x \underline{\mathrm{o}} z) .
$$

Definition 2.5 ([16]). A non-empty subset $T$ of an LA-semihypergroup $\mathbf{H}$ is called sub-LA-semihypergroup of $\mathbf{H}$ if $t_{1} \underline{\mathrm{o}} t_{2} \subseteq T$ for every $t_{1}, t_{2} \in T$.

Definition $2.6([30])$. A sub-LA-semihypergroup $I$ is said to be an interior-hyperideal of $\mathbf{H}$ if $(\mathbf{H} \underline{\mathrm{o}} I) \underline{\mathrm{o}} \mathbf{H} \subseteq I$.

Definition 2.7 ([30]). Let $\mathbf{H}$ be an LA-semihypergroup, then a non-empty subset $A$ of $\mathbf{H}$ is called semiprime if for any $a \in \mathbf{H}$ such that $a \underline{\mathrm{o}} a \subseteq A$ implies $a \in A$. 


\section{Soft SET}

Definition $3.1([8,24])$. A soft set $\mathcal{F}_{A}$ over $\mathcal{U}$ is a set defined by $\mathcal{F}_{A}: E \rightarrow P(\mathcal{U})$ such that $\mathcal{F}_{A}(x)=\emptyset$ if $x \notin A$.

Here $\mathcal{F}_{A}$ is also called an approximate function. A soft set over $\mathcal{U}$ can be represented by the set of ordered pairs

$$
\mathcal{F}_{A}=\left\{\left(x, \mathcal{F}_{A}(x)\right): x \in E, \mathcal{F}_{A}(x) \in P(\mathcal{U})\right\} .
$$

It is clear that a soft set is a parameterized family of subsets of the set $\mathcal{U}$.

Definition 3.2 ([8]). Let $\mathcal{F}_{A}, \mathcal{F}_{B} \in \mathbf{H}(\mathcal{U})$. Then, $\mathcal{F}_{A}$ is called a soft subset of $\mathcal{F}_{B}$ and denoted by $\mathcal{F}_{A} \sqsubseteq \mathcal{F}_{B}$, if $\mathcal{F}_{A}(x) \subseteq \mathcal{F}_{B}(x)$ for all $x \in E$.

Definition $3.3([8])$. Let $\mathcal{F}_{A}, \mathcal{F}_{B} \in \mathbf{H}(\mathcal{U})$. Then, union of $\mathcal{F}_{A}$ and $\mathcal{F}_{B}$ denoted by $\mathcal{F}_{A} \widetilde{\cup} \mathcal{F}_{B}$, is defined as $\mathcal{F}_{A} \widetilde{\cup} \mathcal{F}_{B}=\mathcal{F}_{A \widetilde{\cup} B}$, where $\mathcal{F}_{A \widetilde{\cup} B}(x)=\mathcal{F}_{A}(x) \cup \mathcal{F}_{B}(x)$ for all $x \in E$.

Definition $3.4([8])$. Let $\mathcal{F}_{A}, \mathcal{F}_{B} \in \mathbf{H}(\mathcal{U})$. Then, intersection of $\mathcal{F}_{A}$ and $\mathcal{F}_{B}$ denoted by $\mathcal{F}_{A} \widetilde{\cap}_{\mathcal{F}}$, is defined as $\mathcal{F}_{A} \widetilde{\cap}^{\mathcal{F}_{B}}=\mathcal{F}_{A \widetilde{\cap} B}$, where $\mathcal{F}_{A \widetilde{\cap} B}(x)=\mathcal{F}_{A}(x) \cap \mathcal{F}_{B}(x)$ for all $x \in E$.

Definition $3.5([21])$. Let $Y$ be a subset of $\mathbf{H}$. We denote the soft characteristic function of $Y$ by $\mathcal{H}_{Y}$ and is defined as:

$$
\mathcal{H}_{Y}(y)=\left\{\begin{aligned}
\mathcal{U}, & \text { if } y \in Y \\
\emptyset, & \text { if } y \notin Y
\end{aligned}\right.
$$

In this paper, we denote an LA-semihypergroup $\mathbf{H}$ as a set of parameters.

Let $\mathbf{H}$ be an LA-semihypergroup. For $x \in \mathbf{H}$, we define $\mathbb{H}_{x}=\{(y, z) \in \mathbf{H} \times \mathbf{H}$ : $x \in y \underline{\mathrm{O}} z\}$.

Definition 3.6 ([21]). Let $\mathcal{F}_{\mathbf{H}}$ and $\mathcal{G}_{\mathbf{H}}$ be two soft sets of an LA-semihypergroup $\mathbf{H}$ over $\mathcal{U}$. Then, the soft product $\mathcal{F}_{\mathbf{H}} \hat{\diamond} \mathcal{G}_{\mathbf{H}}$ is a soft set of $\mathbf{H}$ over $\mathcal{U}$, defined by

$$
\left(\mathcal{F}_{\mathbf{H}} \hat{\diamond} \mathcal{G}_{\mathbf{H}}\right)(x)= \begin{cases}\bigcup_{(y, z) \in \mathbb{H}_{x}}\left\{\mathcal{F}_{\mathbf{H}}(y) \cap \mathcal{G}_{\mathbf{H}}(z)\right\}, & \text { if } \mathbb{H}_{x} \neq \emptyset, \\ \emptyset, & \text { if } \mathbb{H}_{x}=\emptyset,\end{cases}
$$

for all $x \in \mathbf{H}$.

Theorem 3.1 ([21]). Let $X$ and $Y$ be non-empty subsets of an LA-semihypergroup $\mathbf{H}$. Then

(1) If $X \subseteq Y$, then $\mathcal{H}_{X} \sqsubseteq \mathcal{H}_{Y}$;

(2) $\mathcal{H}_{X} \widetilde{\cap} \mathcal{H}_{Y}=\mathcal{H}_{X \cap Y}, \mathcal{H}_{X} \widetilde{\cup} \mathcal{H}_{Y}=\mathcal{H}_{X \cup Y}$;

(3) $\mathcal{H}_{X} \hat{\diamond} \mathcal{H}_{Y}=\mathcal{H}_{X} \underline{o} Y$.

Definition 3.7 ([21]). A non-null soft set $\mathcal{F}_{\mathbf{H}}$ is said to be an S.I. sub-LA-semihypergroup of $\mathbf{H}$ over $\mathcal{U}$ if

$$
\bigcap_{\vartheta \in x \underline{\underline{O}} y} \mathcal{F}_{\mathbf{H}}(\vartheta) \supseteq \mathcal{F}_{\mathbf{H}}(x) \cap \mathcal{F}_{\mathbf{H}}(y), \quad \text { for all } x, y \in \mathbf{H} .
$$


Definition 3.8. An S.I. sub-LA-semihypergroup $\mathcal{F}_{\mathbf{H}}$ is said to be an S.I. bi-hyperideal of $\mathbf{H}$ over $\mathcal{U}$ if

$$
\bigcap_{\vartheta \in(x \underline{\underline{O}} y) \underline{\mathrm{O}} z} \mathcal{F}_{\mathbf{H}}(\vartheta) \supseteq \mathcal{F}_{\mathbf{H}}(x) \cap \mathcal{F}_{\mathbf{H}}(z), \quad \text { for all } x, y, z \in \mathbf{H} .
$$

Theorem 3.2 ([21]). A non-null soft set $\mathcal{F}_{\mathbf{H}}$ is an S.I. sub-LA-semihypergroup of $\mathbf{H}$ over $\mathcal{U}$ if and only if

$$
\mathcal{F}_{\mathbf{H}} \hat{\diamond} \mathcal{F}_{\mathbf{H}} \sqsubseteq \mathcal{F}_{\mathbf{H}} .
$$

Corollary 3.1 ([21]). In an LA-semihypergroup $\mathbf{H}$ with left identity, $\mathcal{H}_{\mathbf{H}} \hat{\diamond} \mathcal{H}_{\mathbf{H}}=\mathcal{H}_{\mathbf{H}}$.

Theorem 3.3 ([21]). Let $\mathbf{H}$ be an LA-semihypergroup and $\mathbf{H}(\mathcal{U})$ be the set of all soft sets of $\mathbf{H}$ over $\mathfrak{U}$. Then $(\mathbf{H}(\mathcal{U}), \hat{\diamond})$ is an LA-semigroup.

Theorem 3.4 ([21]). If $\mathbf{H}$ is an LA-semihypergroup. Then medial law holds in $\mathbf{H}(\mathcal{U})$.

Theorem 3.5 ([21]). Let $\mathbf{H}$ be an LA-semihypergroup with left identity and $\mathcal{F}_{\mathbf{H}}, \mathcal{G}_{\mathbf{H}}, \mathcal{K}_{\mathbf{H}}$, $\mathcal{L}_{\mathbf{H}} \in \mathbf{H}(\mathcal{U})$. Then following holds:

(i) $\mathcal{F}_{\mathbf{H}} \hat{\diamond}\left(\mathcal{G}_{\mathbf{H}} \hat{\diamond} \mathcal{K}_{\mathbf{H}}\right)=\mathcal{G}_{\mathbf{H}} \hat{\diamond}\left(\mathcal{F}_{\mathbf{H}} \hat{\diamond} \mathcal{K}_{\mathbf{H}}\right)$;

(ii) $\left(\mathcal{F}_{\mathbf{H}} \hat{\diamond} \mathcal{G}_{\mathbf{H}}\right) \hat{\diamond}\left(\mathcal{K}_{\mathbf{H}} \hat{\diamond} \mathcal{L}_{\mathbf{H}}\right)=\left(\mathcal{L}_{\mathbf{H}} \hat{\diamond} \mathcal{K}_{\mathbf{H}}\right) \hat{\diamond}\left(\mathcal{G}_{\mathbf{H}} \hat{\diamond} \mathcal{F}_{\mathbf{H}}\right)$.

\section{SOFt Interior-HyperideAls IN LA-SEMiHypergroups}

In this section, we define soft interior-hyperideals in LA-semihypergroups and establish some of their elementary properties.

Definition 4.1. An S.I. sub-LA-semihypergroup $\mathcal{F}_{\mathbf{H}}$ is said to be an S.I. interiorhyperideal of $\mathbf{H}$ over $\mathcal{U}$ if

$$
\bigcap_{\vartheta \in(x \underline{\mathrm{O}} y) \underline{\mathrm{O}} z} \mathcal{F}_{\mathbf{H}}(\vartheta) \supseteq \mathcal{F}_{\mathbf{H}}(y), \quad \text { for all } x, y, z \in \mathbf{H} .
$$

Example 4.1. An insurance company offers on some insurances to its agents defined in a set $\mathbf{H}=\{$ Health Insurance (Hlth. Ins.), Home Insurance (Hme. Ins.), Property Insurance (Prop. Ins.), Vehicle Insurance (V.I.), Computer Insurance (C.I.) $\}$ with the composition Table 1.

TABLE 1.

\begin{tabular}{c||ccccc}
$\underline{\mathrm{o}}$ & Health Ins. & Home Ins. & Prop. Ins. & V. I. & C. I. \\
\hline Health Ins. & Health Ins. & Home Ins. & Prop. Ins. & V. I. & C. I. \\
Home Ins. & Prop. Ins. & V. I. & V. I. & \{V. I., C. I. $\}$ & C.I. \\
Prop. Ins. & Health Ins. & V. I. & V. I. & $\{$ V. I., C. I. $\}$ & C.I. \\
V. I. & V. I. & \{V. I., C. I. $\}$ & \{V. I., C. I. & $\{$ V. I., C. I. $\}$ & C. I. \\
C. I. & C. I. & C. I. & C. I. & C. I. & C. I.
\end{tabular}


Let $A=$ 'Husband' and $B=$ 'Wife'. Then the hyperoperation defined in the above composition table as: $(x \underline{\mathrm{o}} y)=$ if the agent does $x$ insurance of $A$ and $y$ insurance of $B$, then he will get $X$ insurances free of cost, where $x, y \in \mathbf{H}$ and $X \subseteq \mathbf{H}$. Therefore, $(\mathbf{H}, \underline{\text { o }})$ will be an LA-semihypergroup.

Now, let $\mathcal{U}=\left\{A_{1}, A_{2}, A_{3}, A_{4}, A_{5}\right\}$ be the set of agents who does insurances to husbands and their wives. Define a soft set $\mathcal{F}_{\mathbf{H}}: \mathbf{H} \rightarrow P(\mathcal{U})$ by

$\mathcal{F}_{\mathbf{H}}($ Health Ins. $)=\left\{A_{1}, A_{2}\right\}$, means the agents who got a health insurance free,

$\mathcal{F}_{\mathbf{H}}$ (Home Ins.) $=\left\{A_{1}, A_{2}\right\}$, means the agents who got a home insurance free, $\mathcal{F}_{\mathbf{H}}$ (Prop. Ins.) $=\left\{A_{1}, A_{2}, A_{3}\right\}$, means the agents who got a property insurance free, $\mathcal{F}_{\mathbf{H}}(\mathrm{V}$. I. $)=\left\{A_{1}, A_{2}, A_{3}, A_{4}\right\}$, means the agents who got a vehicle insurance free and $\mathcal{F}_{\mathbf{H}}(\mathrm{C}$. I. $)=\left\{A_{1}, A_{2}, A_{3}, A_{4}, A_{5}\right\}$, means the agents who got a computer insurance free.

Then, we can verify that $\bigcap_{\vartheta \in(x \underline{\mathrm{O}} y) \underline{\mathrm{O}} z} \mathcal{F}_{\mathbf{H}}(\vartheta) \supseteq \mathcal{F}_{\mathbf{H}}(y)$ for all $x, y, z \in \mathbf{H}$. Therefore, $\mathcal{F}_{\mathbf{H}}$ is an S.I. interior-hyperideal of $\mathbf{H}$ over $\mathcal{U}$.

Theorem 4.1. If $\mathcal{F}_{\mathbf{H}}$ and $\mathcal{G}_{\mathbf{H}}$ are two S.I. interior-hyperideals of $\mathbf{H}$ over $\mathcal{U}$. Then $\mathcal{F}_{\mathbf{H}} \widetilde{\cap}_{\mathbf{H}}$ is also an S.I. interior-hyperideal of $\mathbf{H}$ over $\mathcal{U}$.

Proof. Assume that $\mathcal{F}_{\mathbf{H}}$ and $\mathcal{G}_{\mathbf{H}}$ are two S.I. interior-hyperideals of $\mathbf{H}$ over $\mathcal{U}$. Then, we have

$$
\begin{aligned}
\left(\mathcal{F}_{\mathbf{H}} \widetilde{\bigcap} \mathcal{G}_{\mathbf{H}}\right) \hat{\diamond}\left(\mathcal{F}_{\mathbf{H}} \widetilde{\bigcap} \mathcal{G}_{\mathbf{H}}\right) & \sqsubseteq \mathcal{F}_{\mathbf{H}} \hat{\diamond} \mathcal{F}_{\mathbf{H}} \\
& \sqsubseteq \mathcal{F}_{\mathbf{H}} .
\end{aligned}
$$

In a similar way, $\left(\mathcal{F}_{\mathbf{H}} \widetilde{\cap} \mathcal{G}_{\mathbf{H}}\right) \hat{\diamond}\left(\mathcal{F}_{\mathbf{H}} \widetilde{\cap} \mathcal{G}_{\mathbf{H}}\right) \sqsubseteq \mathcal{G}_{\mathbf{H}}$. It implies $\left(\mathcal{F}_{\mathbf{H}} \widetilde{\cap} \mathcal{G}_{\mathbf{H}}\right) \hat{\diamond}\left(\mathcal{F}_{\mathbf{H}} \widetilde{\cap} \mathcal{G}_{\mathbf{H}}\right)$ $\sqsubseteq\left(\mathcal{F}_{\mathbf{H}} \widetilde{\cap} \mathcal{G}_{\mathbf{H}}\right)$. Also, we have

$$
\begin{aligned}
\left(\mathcal{H}_{\mathbf{H}} \hat{\diamond}\left(\mathcal{F}_{\mathbf{H}} \widetilde{\bigcap} \mathcal{G}_{\mathbf{H}}\right)\right) \hat{\diamond} \mathcal{H}_{\mathbf{H}} \sqsubseteq\left(\mathcal{H}_{\mathbf{H}} \hat{\diamond} \mathcal{F}_{\mathbf{H}}\right) \hat{\diamond} \mathcal{H}_{\mathbf{H}} \\
\\
\sqsubseteq \mathcal{F}_{\mathbf{H}} .
\end{aligned}
$$

In a similar way, $\left(\mathcal{H}_{\mathbf{H}} \hat{\diamond}\left(\mathcal{F}_{\mathbf{H}} \widetilde{\cap} \mathcal{G}_{\mathbf{H}}\right)\right) \hat{\diamond} \mathcal{H}_{\mathbf{H}} \sqsubseteq \mathcal{G}_{\mathbf{H}}$. Therefore, $\left(\mathcal{H}_{\mathbf{H}} \hat{\diamond}\left(\mathcal{F}_{\mathbf{H}} \widetilde{\cap} \mathcal{G}_{\mathbf{H}}\right)\right) \hat{\diamond} \mathcal{H}_{\mathbf{H}}$ $\sqsubseteq \mathcal{F}_{\mathbf{H}} \widetilde{\cap} \mathcal{G}_{\mathbf{H}}$. Hence, $\mathcal{F}_{\mathbf{H}} \widetilde{\cap} \mathcal{G}_{\mathbf{H}}$ is an S.I. interior-hyperideal of $\mathbf{H}$ over $\mathcal{U}$.

Theorem 4.2. Let $X$ be any non-empty subset of an LA-semihypergroup $\mathbf{H}$. Then $X$ is an interior-hyperideal of $\mathbf{H}$ if and only if $\mathcal{H}_{X}$ is an S.I. interior-hyperideal of $\mathbf{H}$ over U.

Proof. Proof is easy, hence omitted.

Theorem 4.3. An S.I. sub-LA-semihypergroup $\mathcal{F}_{\mathbf{H}}$ is an S.I. interior-hyperideal of $\mathbf{H}$ over $\mathcal{U}$ if and only if

$$
\left(\mathcal{H}_{\mathbf{H}} \hat{\diamond} \mathcal{F}_{\mathbf{H}}\right) \hat{\diamond} \mathcal{H}_{\mathbf{H}} \sqsubseteq \mathcal{F}_{\mathbf{H}} .
$$

Proof. Assume that $\mathcal{F}_{\mathbf{H}}$ is an S.I. interior-hyperideal of $\mathbf{H}$ over $\mathcal{U}$, then

$$
\bigcap_{\vartheta \in(x \underline{\mathrm{O}} y) \underline{\mathrm{O}} z} \mathcal{F}_{\mathbf{H}}(\vartheta) \supseteq \mathcal{F}_{\mathbf{H}}(y), \quad \text { for all } x, y, z \in \mathbf{H} .
$$


Now, if $\mathbb{H}_{x}=\emptyset$, then $\left(\mathcal{H}_{\mathbf{H}} \hat{\diamond} \mathcal{F}_{\mathbf{H}} \hat{\diamond} \mathcal{H}_{\mathbf{H}}\right)(x)=\emptyset$. Thus, it would yield $\left(\mathcal{H}_{\mathbf{H}} \hat{\diamond} \mathcal{F}_{\mathbf{H}} \hat{\diamond} \mathcal{H}_{\mathbf{H}}\right)(x) \subseteq \mathcal{F}_{\mathbf{H}}(x)$. Therefore, $\left(\mathcal{H}_{\mathbf{H}} \hat{\diamond} \mathcal{F}_{\mathbf{H}}\right) \hat{\diamond} \mathcal{H}_{\mathbf{H}} \sqsubseteq \mathcal{F}_{\mathbf{H}}$.

If $\mathbb{H}_{x} \neq \emptyset$, then there exists $u, v, p, q \in \mathbf{H}$ such that $x \in u \underline{\mathrm{o}} v$ and $u \in p \underline{\mathrm{o}} q$. So, $(u, v) \in \mathbb{H}_{x}$ and $(p, q) \in \mathbb{H}_{u}$. Thus, we have

$$
\begin{aligned}
& \left(\left(\mathcal{H}_{\mathbf{H}} \hat{\diamond} \mathcal{F}_{\mathbf{H}}\right) \hat{\diamond} \mathcal{H}_{\mathbf{H}}\right)(x)=\bigcup_{(u, v) \in \mathbb{H}_{x}}\left[\left(\mathcal{H}_{\mathbf{H}} \hat{\diamond} \mathcal{F}_{\mathbf{H}}\right)(u) \bigcap \mathcal{H}_{\mathbf{H}}(v)\right] \\
& =\bigcup_{x \in u \underline{\mathrm{O}} v}\left[\bigcup_{(p, q) \in \mathbb{H}_{u}}\left(\mathcal{H}_{\mathbf{H}}(p) \bigcap \mathcal{F}_{\mathbf{H}}(q)\right) \bigcap \mathcal{H}_{\mathbf{H}}(v)\right] \\
& =\bigcup_{x \in u \underline{\mathrm{O}} v}\left[\bigcup_{u \in p \underline{\mathrm{O}} q}\left(\mathcal{H}_{\mathbf{H}}(p) \bigcap \mathcal{F}_{\mathbf{H}}(q)\right) \bigcap \mathcal{U}\right] \\
& =\bigcup_{x \in u \underline{\mathrm{O}} v}\left[\bigcup_{u \in p \underline{\underline{\mathrm{O}} q}}\left(\mathcal{H}_{\mathbf{H}}(p) \bigcap \mathcal{F}_{\mathbf{H}}(q)\right)\right] \\
& =\bigcup_{x \in u \underline{\mathrm{O}} v}\left[\bigcup_{u \in p \underline{\mathrm{O} q}}\left(\mathcal{U} \cap \mathcal{F}_{\mathbf{H}}(q)\right)\right] \\
& =\bigcup_{x \in u \underline{\mathrm{O}} v}\left[\bigcup_{u \in p \underline{\mathrm{O}} q}\left(\mathcal{F}_{S}(q)\right)\right] \\
& =\bigcup_{x \in(p \underline{\mathrm{O}} q) \underline{\mathrm{O}} v}\left(\mathcal{F}_{\mathbf{H}}(q)\right) \\
& \subseteq \bigcup_{x \in(p \underline{\mathrm{O}} q) \underline{\mathrm{O}} v}\left\{\bigcap_{\vartheta \in(r \underline{\mathrm{O}} q) \underline{\mathrm{O}} t} \mathcal{F}_{\mathbf{H}}(\vartheta)\right\}, \\
& \text { (as } \mathcal{F}_{\mathbf{H}} \text { is an S.I. interior hyperideal) } \\
& \subseteq \bigcup_{x \in(p \underline{\mathrm{O}} q) \underline{\mathrm{O}} v}\left\{\bigcap_{x \in(r \underline{\mathrm{O}} q) \underline{\mathrm{O}} t} \mathcal{F}_{\mathbf{H}}(x)\right\} \\
& \subseteq \bigcup_{x \in(p \underline{O} q) \underline{O} v}\left\{\bigcap_{x \in(p \underline{O} q) \underline{O} v} \mathcal{F}_{\mathbf{H}}(x)\right\} \\
& =\mathcal{F}_{\mathbf{H}}(x) \text {. }
\end{aligned}
$$

Hence, $\left(\mathcal{H}_{\mathbf{H}} \hat{\diamond} \mathcal{F}_{\mathbf{H}}\right) \hat{\diamond} \mathcal{H}_{\mathbf{H}} \sqsubseteq \mathcal{F}_{\mathbf{H}}$.

Conversely, suppose that $\left(\mathcal{H}_{\mathbf{H}} \hat{\diamond} \mathcal{F}_{\mathbf{H}}\right) \hat{\diamond} \mathcal{H}_{\mathbf{H}} \sqsubseteq \mathcal{F}_{\mathbf{H}}$. Now to show $\mathcal{F}_{\mathbf{H}}$ is an S.I. interiorhyperideal of $\mathbf{H}$ over $\mathcal{U}$, we have

$$
\begin{aligned}
\bigcap_{\vartheta \in(x \underline{\mathrm{O}} y) \underline{\mathrm{O}} z} \mathcal{F}_{\mathbf{H}}(\vartheta) & \left.\supseteq \bigcap_{\vartheta \in(x} \underline{\mathrm{O}} y\right) \underline{\mathrm{O}} z \\
& =\bigcap_{\vartheta \in(x} \underline{\left.\bigcap_{y} y\right) \underline{\mathrm{O}} z}\left(\left(\mathcal{H}_{\mathbf{H}} \hat{\diamond} \mathcal{F}_{\mathbf{H}} \hat{\diamond} \mathcal{H}_{\mathbf{H}}\right)(\vartheta)\right. \\
& \left.=\bigcap_{\vartheta \in(x} \underline{\mathrm{O}} y\right) \underline{\mathrm{O}} z
\end{aligned}
$$




$$
\begin{aligned}
& =\bigcap_{\vartheta \in(x \underline{\mathrm{O}} y) \underline{\mathrm{O}} z}\left\{\bigcup_{\vartheta \in u \underline{\mathrm{O}} v}\left[\bigcup_{(p, q) \in \mathbb{H}_{u}}\left(\mathcal{H}_{\mathbf{H}}(p) \bigcap \mathcal{F}_{\mathbf{H}}(q)\right) \bigcap \mathcal{H}_{\mathbf{H}}(v)\right]\right\} \\
& =\bigcap_{\vartheta \in(x \underline{\mathrm{O}} y) \underline{\underline{\mathrm{O}} z}}\left\{\bigcup_{\vartheta \in u \underline{\mathrm{O}} v}\left[\bigcup_{u \in p \underline{\mathrm{O}} q}\left(\mathcal{H}_{\mathbf{H}}(p) \bigcap \mathcal{F}_{\mathbf{H}}(q)\right) \bigcap \mathcal{U}\right]\right\} \\
& =\bigcap_{\vartheta \in(x \underline{\mathrm{O}} y) \underline{\mathrm{O}} z}\left\{\bigcup_{\vartheta \in u \underline{\mathrm{O}} v}\left[\bigcup_{u \in p \underline{\mathrm{O} q}}\left(\mathcal{H}_{\mathbf{H}}(p) \bigcap \mathcal{F}_{\mathbf{H}}(q)\right)\right]\right\} \\
& =\bigcap_{\vartheta \in(x \underline{\mathrm{O}} y) \underline{\mathrm{O}} z}\left\{\bigcup_{\vartheta \in u \underline{\mathrm{O}} v}\left[\bigcup_{u \in p \underline{\mathrm{O}} q}\left(\mathcal{U} \cap \mathcal{F}_{\mathbf{H}}(q)\right)\right]\right\} \\
& =\bigcap_{\vartheta \in(x \underline{\mathrm{O}} y) \underline{\mathrm{O}} z}\left\{\bigcup_{\vartheta \in u \underline{\mathrm{O}} v}\left[\bigcup_{u \in p \underline{\mathrm{O}} q}\left(\mathcal{F}_{\mathbf{H}}(q)\right)\right]\right\} \\
& \supseteq \bigcap_{\vartheta \in(x \underline{\mathrm{O}} y) \underline{\mathrm{O}} z}\left\{\bigcup_{\vartheta \in u \underline{\mathrm{O}} v}\left[\bigcup_{u \in x \underline{\mathrm{O}} y}\left(\mathcal{F}_{\mathbf{H}}(y)\right)\right]\right\} \\
& \supseteq \bigcap_{\vartheta \in(x \underline{\mathrm{O}} y) \underline{\mathrm{O}} z}\left\{\bigcup_{\vartheta \in u \underline{\mathrm{O}} z}\left[\bigcup_{u \in x \underline{\mathrm{O}} y}\left(\mathcal{F}_{\mathbf{H}}(y)\right)\right]\right\} \\
& =\bigcap_{\vartheta \in(x \underline{\mathrm{O}} y) \underline{\mathrm{O}} z}\left\{\bigcup_{\vartheta \in(x \underline{\mathrm{O}} y) \underline{\mathrm{O}} z}\left(\mathcal{F}_{\mathbf{H}}(y)\right)\right\} \\
& =\mathcal{F}_{\mathbf{H}}(y) \text {. }
\end{aligned}
$$

It follows that $\mathcal{F}_{\mathbf{H}}$ is an S.I. interior-hyperideal of $\mathbf{H}$ over $\mathcal{U}$.

Theorem 4.4. If $\mathcal{F}_{\mathbf{H}}$ and $\mathcal{G}_{\mathbf{H}}$ are S.I. interior-hyperideals of $\mathbf{H}$ over $\mathfrak{U}$ with left identity. Then the S.I. product $\mathcal{F}_{\mathbf{H}} \hat{\diamond} \mathcal{G}_{\mathbf{H}}$ is an S.I. interior-hyperideal of $\mathbf{H}$ over $\mathcal{U}$.

Proof. Let $\mathcal{F}_{\mathbf{H}}$ and $\mathcal{G}_{\mathbf{H}}$ be S.I. interior-hyperideals of $\mathbf{H}$ over $\mathcal{U}$ with left identity. Then, we have

$$
\left(\mathcal{F}_{\mathbf{H}} \hat{\diamond} \mathcal{G}_{\mathbf{H}}\right) \hat{\diamond}\left(\mathcal{F}_{\mathbf{H}} \hat{\diamond} \mathcal{G}_{\mathbf{H}}\right)=\left(\mathcal{F}_{\mathbf{H}} \hat{\diamond} \mathcal{F}_{\mathbf{H}}\right) \hat{\diamond}\left(\mathcal{G}_{\mathbf{H}} \hat{\diamond} \mathcal{G}_{\mathbf{H}}\right) \sqsubseteq \mathcal{F}_{\mathbf{H}} \hat{\diamond} \mathcal{G}_{\mathbf{H}}
$$

It implies $\mathcal{F}_{\mathbf{H}} \hat{\diamond} \mathcal{G}_{\mathbf{H}}$ is an S.I. sub-LA-semihypergroup of $\mathbf{H}$ over $\mathcal{U}$. Also, we have

$$
\begin{aligned}
\left(\mathcal{H}_{\mathbf{H}} \hat{\diamond}\left(\mathcal{F}_{\mathbf{H}} \hat{\diamond} \mathcal{G}_{\mathbf{H}}\right)\right) \hat{\diamond} \mathcal{H}_{\mathbf{H}} & =\left(\left(\mathcal{H}_{\mathbf{H}} \hat{\diamond} \mathcal{H}_{\mathbf{H}}\right) \hat{\diamond}\left(\mathcal{F}_{\mathbf{H}} \hat{\diamond} \mathcal{G}_{\mathbf{H}}\right)\right) \hat{\diamond} \mathcal{H}_{\mathbf{H}} \\
& =\left(\left(\mathcal{H}_{\mathbf{H}} \hat{\diamond} \mathcal{F}_{\mathbf{H}}\right) \hat{\diamond}\left(\mathcal{H}_{\mathbf{H}} \hat{\diamond} \mathcal{G}_{\mathbf{H}}\right)\right) \hat{\diamond} \mathcal{H}_{\mathbf{H}} \\
& =\left(\left(\mathcal{H}_{\mathbf{H}} \hat{\diamond} \mathcal{F}_{\mathbf{H}}\right) \hat{\diamond}\left(\mathcal{H}_{\mathbf{H}} \hat{\diamond} \mathcal{G}_{\mathbf{H}}\right)\right) \hat{\diamond}\left(\mathcal{H}_{\mathbf{H}} \hat{\diamond} \mathcal{H}_{\mathbf{H}}\right) \\
& =\left(\left(\mathcal{H}_{\mathbf{H}} \hat{\diamond} \mathcal{F}_{\mathbf{H}}\right) \hat{\diamond} \mathcal{H}_{\mathbf{H}}\right) \hat{\diamond}\left(\left(\mathcal{H}_{\mathbf{H}} \hat{\diamond} \mathcal{G}_{\mathbf{H}}\right) \hat{\diamond} \mathcal{H}_{\mathbf{H}}\right) \\
& \sqsubseteq \mathcal{F}_{\mathbf{H}} \hat{\diamond} \mathcal{G}_{\mathbf{H}} .
\end{aligned}
$$

This shows that $\mathcal{F}_{\mathbf{H}} \hat{\diamond} \mathcal{G}_{\mathbf{H}}$ is an S.I. interior-hyperideal of $\mathbf{H}$ over $\boldsymbol{U}$.

Definition 4.2. A soft set $\mathcal{F}_{\mathbf{H}}$ of an LA-semihypergroup $\mathbf{H}$ over $\mathcal{U}$ is said to be idempotent if $\mathcal{F}_{\mathbf{H}} \hat{\diamond} \mathcal{F}_{\mathbf{H}}=\mathcal{F}_{\mathbf{H}}$. 
Example 4.2. Consider an LA-semihypergroup given in the Example 4.1. Now, let $\mathcal{U}=\left\{A_{1}, A_{2}, A_{3}, A_{4}, A_{5}\right\}$ be the set of agents who does insurances to husbands and their wives. Define a soft set $\mathcal{F}_{\mathbf{H}}: \mathbf{H} \rightarrow P(\mathcal{U})$ by

$\mathcal{F}_{\mathbf{H}}($ Health Ins. $)=\emptyset$, means the agents who got a health insurance free,

$\mathcal{F}_{\mathbf{H}}$ (Home Ins. $)=\emptyset$, means the agents who got a home insurance free,

$\mathcal{F}_{\mathbf{H}}$ (Prop. Ins.) $=\emptyset$, means the agents who got a property insurance free,

$\mathcal{F}_{\mathbf{H}}$ (V.I.) $=\left\{\mathrm{A}_{1}, \mathrm{~A}_{2}, \mathrm{~A}_{3}\right\}$, means the agents who got a vehicle insurance free and

$\mathcal{F}_{\mathbf{H}}$ (C.I.) $=\left\{\mathrm{A}_{1}, \mathrm{~A}_{2}, \mathrm{~A}_{3}\right\}$, means the agents who got a computer insurance free.

Then, we can easily verify that $\mathcal{F}_{\mathbf{H}} \hat{\diamond} \mathcal{F}_{\mathbf{H}}=\mathcal{F}_{\mathbf{H}}$. Hence, $\mathcal{F}_{\mathbf{H}}$ is idempotent.

Proposition 4.1. Every idempotent S.I. bi-hyperideal of $\mathbf{H}$ over $\mathfrak{U}$ with left identity is an S.I. interior-hyperideal of $\mathbf{H}$ over $\mathcal{U}$.

Proof. Assume that $\mathcal{F}_{\mathbf{H}}$ is an idempotent S.I. bi-hyperideal of $\mathbf{H}$ over $\mathcal{U}$, then $\left(\mathcal{F}_{\mathbf{H}} \hat{\diamond} \mathcal{H}_{\mathbf{H}}\right) \hat{\diamond} \mathcal{F}_{\mathbf{H}} \sqsubseteq \mathcal{F}_{\mathbf{H}}$. Thus, we have

$$
\begin{aligned}
\left(\mathcal{H}_{\mathbf{H}} \hat{\diamond} \mathcal{F}_{\mathbf{H}}\right) \hat{\diamond} \mathcal{H}_{\mathbf{H}} & =\left(\mathcal{H}_{\mathbf{H}} \hat{\diamond}\left(\mathcal{F}_{\mathbf{H}} \hat{\diamond} \mathcal{F}_{\mathbf{H}}\right)\right) \hat{\diamond}\left(\mathcal{H}_{\mathbf{H}} \hat{\diamond} \mathcal{H}_{\mathbf{H}}\right) \\
& =\left(\mathcal{H}_{\mathbf{H}} \hat{\diamond} \mathcal{H}_{\mathbf{H}}\right) \hat{\diamond}\left(\left(\mathcal{F}_{\mathbf{H}} \hat{\diamond} \mathcal{F}_{\mathbf{H}}\right) \hat{\diamond} \mathcal{H}_{\mathbf{H}}\right) \\
& =\mathcal{H}_{\mathbf{H}} \hat{\diamond}\left(\left(\mathcal{F}_{\mathbf{H}} \hat{\diamond} \mathcal{F}_{\mathbf{H}}\right) \hat{\diamond} \mathcal{H}_{\mathbf{H}}\right) \\
& =\left(\mathcal{F}_{\mathbf{H}} \hat{\diamond} \mathcal{F}_{\mathbf{H}}\right) \hat{\diamond}\left(\mathcal{H}_{\mathbf{H}} \hat{\diamond} \mathcal{H}_{\mathbf{H}}\right) \\
& =\left(\left(\mathcal{H}_{\mathbf{H}} \hat{\diamond} \mathcal{H}_{\mathbf{H}}\right) \hat{\diamond} \mathcal{F}_{\mathbf{H}}\right) \hat{\diamond} \mathcal{F}_{\mathbf{H}} \\
& =\left(\left(\mathcal{H}_{\mathbf{H}} \hat{\diamond} \mathcal{H}_{\mathbf{H}}\right) \hat{\diamond}\left(\mathcal{F}_{\mathbf{H}} \hat{\diamond} \mathcal{F}_{\mathbf{H}}\right)\right) \hat{\diamond} \mathcal{F}_{\mathbf{H}} \\
& =\left(\left(\mathcal{F}_{\mathbf{H}} \hat{\diamond} \mathcal{F}_{\mathbf{H}}\right) \hat{\diamond}\left(\mathcal{H}_{\mathbf{H}} \hat{\diamond} \mathcal{H}_{\mathbf{H}}\right)\right) \hat{\diamond} \mathcal{F}_{\mathbf{H}} \\
& =\left(\mathcal{F}_{\mathbf{H}} \hat{\diamond} \mathcal{H}_{\mathbf{H}}\right) \hat{\diamond} \mathcal{F}_{\mathbf{H}} \\
& \sqsubseteq \mathcal{F}_{\mathbf{H}} .
\end{aligned}
$$

Hence, $\mathcal{F}_{\mathbf{H}}$ is an S.I. interior-hyperideal of $\mathbf{H}$ over $\mathcal{U}$.

\section{Characterization of Left Regular LA-Semihypergroups}

In this section, we characterize left regular LA-semihypergroup using soft interiorhyperideals.

Definition 5.1. An element $l_{r}$ of an LA-semihypergroup $\mathbf{H}$ is called a left regular element if there exists an element $x \in \mathbf{H}$ such that $l_{r} \in x \underline{\mathrm{o}}\left(l_{r} \underline{\mathrm{o}} l_{r}\right)$. If every element of $\mathbf{H}$ is left regular, then $\mathbf{H}$ is called a left regular LA-semihypergroup.

Lemma 5.1. Let $\mathbf{H}$ be a left regular LA-semihypergroup with left identity. Then for any S.I. interior-hyperideal $\mathcal{F}_{\mathbf{H}}$ of $\mathbf{H}$ over $\mathcal{U}$, we have $\left(\mathcal{H}_{\mathbf{H}} \hat{\diamond} \mathcal{F}_{\mathbf{H}}\right) \hat{\diamond} \mathcal{H}_{\mathbf{H}}=\mathcal{F}_{\mathbf{H}}$.

Proof. Assume that $\mathcal{F}_{\mathbf{H}}$ is an S.I. interior-hyperideal of $\mathbf{H}$ over $\mathcal{U}$, then by the Theorem 4.3, $\left(\mathcal{H}_{\mathbf{H}} \hat{\diamond} \mathcal{F}_{\mathbf{H}}\right) \hat{\diamond} \mathcal{H}_{\mathbf{H}} \sqsubseteq \mathcal{F}_{\mathbf{H}}$. Now, it is only remains to prove that $\left(\mathcal{H}_{\mathbf{H}} \hat{\diamond} \mathcal{F}_{\mathbf{H}}\right) \hat{\diamond} \mathcal{H}_{\mathbf{H}} \sqsupseteq$ 
$\mathcal{F}_{\mathbf{H}}$. By assumption, $\mathbf{H}$ is left regular, thus for any $l_{r} \in \mathbf{H}$, there exists $x \in \mathbf{H}$ such that $l_{r} \in x \underline{\mathrm{o}}\left(l_{r} \underline{\mathrm{o}} l_{r}\right)$. Let $e \in \mathbf{H}$ be the left identity, then we have

$$
\begin{aligned}
l_{r} & \in x \underline{\mathrm{o}}\left(l_{r} \underline{\mathrm{o}} l_{r}\right) \\
& \subseteq(e \underline{\mathrm{o}} x) \underline{\mathrm{o}}\left(l_{r} \underline{\mathrm{o}} l_{r}\right) \\
& =\left(l_{r} \underline{\mathrm{o}} l_{r}\right) \underline{\mathrm{o}}(x \underline{\mathrm{o}} e) \\
& =\left((x \underline{\mathrm{o}} e) \underline{\mathrm{o}} l_{r}\right) \underline{\mathrm{o}} l_{r} \\
& \subseteq\left((x \underline{\mathrm{o}} e) \underline{\mathrm{o}}\left(x \underline{\mathrm{o}}\left(l_{r} \underline{\mathrm{o}} l_{r}\right)\right)\right) \underline{\mathrm{o}} l_{r} \\
& =\left(x \underline{\mathrm{o}}\left((x \underline{\mathrm{o}} e) \underline{\mathrm{o}}\left(l_{r} \underline{\mathrm{o}} l_{r}\right)\right)\right) \underline{\mathrm{o}} l_{r} \\
& =\left(x \underline{\mathrm{o}}\left(\left(l_{r} \underline{\mathrm{o}} l_{r}\right) \underline{\mathrm{o}}(e \underline{\mathrm{o}} x)\right)\right) \underline{\mathrm{o}} l_{r} .
\end{aligned}
$$

It implies there exists $a \in e \underline{\mathrm{O}} x$ such that $l_{r} \in\left(x \underline{\mathrm{o}}\left(\left(l_{r} \underline{\mathrm{O}} l_{r}\right) \underline{\mathrm{o}} a\right)\right) \underline{\mathrm{o}} l_{r}$, there exists $b \in\left(\left(l_{r} \underline{\mathrm{o}} l_{r}\right) \underline{\mathrm{o}} a\right)$ such that $l_{r} \in(x \underline{\mathrm{o}} b) \underline{\mathrm{o}} l_{r}$ and there exists $c \in x \underline{\mathrm{o}} b$ such that $l_{r} \in c \underline{\mathrm{o}} l_{r}$. So, $\left(c, l_{r}\right) \in \mathbb{H}_{l_{r}}$ and $(x, b) \in \mathbb{H}_{c}$. Thus, we have

$$
\begin{aligned}
\left(\left(\mathcal{H}_{\mathbf{H}} \hat{\diamond} \mathcal{F}_{\mathbf{H}}\right) \hat{\diamond} \mathcal{H}_{\mathbf{H}}\right)\left(l_{r}\right) & =\bigcup_{(u, v) \in \mathbb{H}_{l_{r}}}\left[\left(\mathcal{H}_{\mathbf{H}} \hat{\diamond} \mathcal{F}_{\mathbf{H}}\right)(u) \cap \mathcal{H}_{\mathbf{H}}(v)\right] \\
& \supseteq\left(\mathcal{H}_{\mathbf{H}} \hat{\diamond} \mathcal{F}_{\mathbf{H}}\right)(c) \cap \mathcal{H}_{\mathbf{H}}\left(l_{r}\right) \\
& =\left(\mathcal{H}_{\mathbf{H}} \hat{\diamond} \mathcal{F}_{\mathbf{H}}\right)(c) \cap \mathcal{U} \\
& =\bigcup_{(p, q) \in \mathbb{H}_{c}}\left(\mathcal{H}_{\mathbf{H}}(p) \cap \mathcal{F}_{\mathbf{H}}(q)\right) \\
& \supseteq \mathcal{H}_{\mathbf{H}}(x) \cap \mathcal{F}_{\mathbf{H}}(b) \\
& =\mathcal{F}_{\mathbf{H}}(b) .
\end{aligned}
$$

As $\mathcal{F}_{\mathbf{H}}$ is an S.I. interior-hyperideal of $\mathbf{H}$ over $\mathcal{U}$, we have $\left.\bigcap_{\vartheta \in(x} \mathrm{O} y\right)$ o $z \mathcal{F}_{\mathbf{H}}(\vartheta) \supseteq \mathcal{F}_{\mathbf{H}}(y)$ for all $x, y, z \in \mathbf{H}$. Since $b \in\left(l_{r} \underline{\mathrm{o}} l_{r}\right) \underline{\mathrm{o}} a$, it would imply that $\mathcal{F}_{\mathbf{H}}(b) \supseteq \mathcal{F}_{\mathbf{H}}\left(l_{r}\right)$. Therefore, from equation (5.1), we have

$$
\begin{aligned}
\left(\left(\mathcal{H}_{\mathbf{H}} \hat{\diamond} \mathcal{F}_{\mathbf{H}}\right) \hat{\diamond} \mathcal{H}_{\mathbf{H}}\right)\left(l_{r}\right) & \supseteq \mathcal{F}_{\mathbf{H}}(b) \\
& =\mathcal{F}_{\mathbf{H}}\left(l_{r}\right) .
\end{aligned}
$$

Hence, $\left(\mathcal{H}_{\mathbf{H}} \hat{\diamond} \mathcal{F}_{\mathbf{H}}\right) \hat{\diamond} \mathcal{H}_{\mathbf{H}}=\mathcal{F}_{\mathbf{H}}$.

Lemma 5.2. If $\mathbf{H}$ is a left regular LA-semihypergroup with left identity. Then for every S.I. interior-hyperideal $\mathcal{F}_{\mathbf{H}}$ of $\mathbf{H}$ over $\mathcal{U}$, we have

$$
\mathcal{H}_{\mathbf{H}} \hat{\diamond} \mathcal{F}_{\mathbf{H}}=\mathcal{F}_{\mathbf{H}}=\mathcal{F}_{\mathbf{H}} \hat{\diamond} \mathcal{H}_{\mathbf{H}} \text {. }
$$

Proof. Let $\mathcal{F}_{\mathbf{H}}$ be an S.I. interior-hyperideal of a left regular LA-semihypergroup $\mathbf{H}$ over $\mathcal{U}$ with left identity. By Lemma 5.1, $\left(\mathcal{H}_{\mathbf{H}} \hat{\diamond} \mathcal{F}_{\mathbf{H}}\right) \hat{\diamond} \mathcal{H}_{\mathbf{H}}=\mathcal{F}_{\mathbf{H}}$. Thus, we have

$$
\begin{aligned}
\mathcal{H}_{\mathbf{H}} \hat{\diamond} \mathcal{F}_{\mathbf{H}} & =\left(\mathcal{H}_{\mathbf{H}} \hat{\diamond} \mathcal{H}_{\mathbf{H}}\right) \hat{\diamond} \mathcal{F}_{\mathbf{H}}=\left(\mathcal{F}_{\mathbf{H}} \hat{\diamond} \mathcal{H}_{\mathbf{H}}\right) \hat{\diamond} \mathcal{H}_{\mathbf{H}}=\left(\mathcal{F}_{\mathbf{H}} \hat{\diamond} \mathcal{H}_{\mathbf{H}}\right) \hat{\diamond}\left(\mathcal{H}_{\mathbf{H}} \hat{\diamond} \mathcal{H}_{\mathbf{H}}\right) \\
& =\left(\mathcal{H}_{\mathbf{H}} \hat{\diamond} \mathcal{H}_{\mathbf{H}}\right) \hat{\diamond}\left(\mathcal{H}_{\mathbf{H}} \hat{\diamond} \mathcal{F}_{\mathbf{H}}\right)=\left(\left(\mathcal{H}_{\mathbf{H}} \hat{\diamond} \mathcal{F}_{\mathbf{H}}\right) \hat{\diamond} \mathcal{H}_{\mathbf{H}}\right) \hat{\diamond} \mathcal{H}_{\mathbf{H}}=\mathcal{F}_{\mathbf{H}} \hat{\diamond} \mathcal{H}_{\mathbf{H}} .
\end{aligned}
$$


Also, we have

$$
\begin{aligned}
\mathcal{F}_{\mathbf{H}} \hat{\diamond} \mathcal{H}_{\mathbf{H}} & =\mathcal{F}_{\mathbf{H}} \hat{\diamond}\left(\mathcal{H}_{\mathbf{H}} \hat{\diamond} \mathcal{H}_{\mathbf{H}}\right)=\mathcal{H}_{\mathbf{H}} \hat{\diamond}\left(\mathcal{F}_{\mathbf{H}} \hat{\diamond} \mathcal{H}_{\mathbf{H}}\right)=\left(\mathcal{H}_{\mathbf{H}} \hat{\diamond} \mathcal{H}_{\mathbf{H}}\right) \hat{\diamond}\left(\mathcal{F}_{\mathbf{H}} \hat{\diamond} \mathcal{H}_{\mathbf{H}}\right) \\
& =\left(\mathcal{H}_{\mathbf{H}} \hat{\diamond} \mathcal{F}_{\mathbf{H}}\right) \hat{\diamond}\left(\mathcal{H}_{\mathbf{H}} \hat{\diamond} \mathcal{H}_{\mathbf{H}}\right)=\left(\mathcal{F}_{\mathbf{H}} \hat{\diamond} \mathcal{H}_{\mathbf{H}}\right) \hat{\diamond}\left(\mathcal{H}_{\mathbf{H}} \hat{\diamond} \mathcal{H}_{\mathbf{H}}\right) \\
& =\left(\left(\mathcal{H}_{\mathbf{H}} \hat{\diamond} \mathcal{H}_{\mathbf{H}}\right) \hat{\diamond} \mathcal{H}_{\mathbf{H}}\right) \hat{\diamond} \mathcal{F}_{\mathbf{H}}=\mathcal{H}_{\mathbf{H}} \hat{\diamond} \mathcal{F}_{\mathbf{H}}
\end{aligned}
$$

and

$$
\mathcal{H}_{\mathbf{H}} \hat{\diamond} \mathcal{F}_{\mathbf{H}}=\left(\mathcal{H}_{\mathbf{H}} \hat{\diamond} \mathcal{H}_{\mathbf{H}}\right) \hat{\diamond} \mathcal{F}_{\mathbf{H}}=\left(\mathcal{F}_{\mathbf{H}} \hat{\diamond} \mathcal{H}_{\mathbf{H}}\right) \hat{\diamond} \mathcal{H}_{\mathbf{H}}=\left(\mathcal{H}_{\mathbf{H}} \hat{\diamond} \mathcal{F}_{\mathbf{H}}\right) \hat{\diamond} \mathcal{H}_{\mathbf{H}}=\mathcal{F}_{\mathbf{H}}
$$

Hence, $\mathcal{H}_{\mathbf{H}} \hat{\diamond} \mathcal{F}_{\mathbf{H}}=\mathcal{F}_{\mathbf{H}}=\mathcal{F}_{\mathbf{H}} \hat{\diamond} \mathcal{H}_{\mathbf{H}}$.

Definition 5.2. A soft set $\mathcal{F}_{\mathbf{H}}$ is said to be soft semiprime if for all $l_{r} \in \mathbf{H}$, $\mathcal{F}_{\mathbf{H}}\left(l_{r}\right) \supseteq \bigcap_{\vartheta \in l_{r}} \underline{\mathrm{o}} l_{r} \mathcal{F}_{\mathbf{H}}(\vartheta)$.

Example 5.1. Consider an LA-semihypergroup given in the Example 4.1. Now, let $\mathcal{U}=\left\{A_{1}, A_{2}, A_{3}, A_{4}, A_{5}\right\}$ be the set of agents who does insurances to husbands and their wives. Define a soft set $\mathcal{F}_{\mathbf{H}}: \mathbf{H} \rightarrow P(\mathcal{U})$ by

$\mathcal{F}_{\mathbf{H}}$ (Health Ins. $)=\left\{A_{1}, A_{2}, A_{3}\right\}$, means the agents who got a health insurance free, $\mathcal{F}_{\mathbf{H}}$ (Home Ins.) $=\left\{A_{1}, A_{2}, A_{3}, A_{4}\right\}$, means the agents who got a home insurance free, $\mathcal{F}_{\mathbf{H}}$ (Prop. Ins.) $=\left\{A_{1}, A_{2}, A_{3}\right\}$, means the agents who got a property insurance free,

$\mathcal{F}_{\mathbf{H}}$ (V.I.) $=\left\{A_{1}, A_{2}\right\}$, means the agents who got a vehicle insurance free and $\mathcal{F}_{\mathbf{H}}$ (C.I.) $=\left\{A_{1}, A_{2}, A_{3}, A_{4}, A_{5}\right\}$, means the agents who got a computer insurance free. Then, we can easily verify that for all $l_{r} \in \mathbf{H}, \mathcal{F}_{\mathbf{H}}\left(l_{r}\right) \supseteq \bigcap_{\vartheta \in l_{r}} \underline{\mathrm{o}} l_{r} \mathcal{F}_{\mathbf{H}}(\vartheta)$. Hence, $\mathcal{F}_{\mathbf{H}}$ is soft semiprime.

Lemma 5.3. Let $\mathbf{H}$ be an LA-semihypergroup. Then $A$ is semiprime if and only if $\mathcal{H}_{A}$ is soft semiprime.

Proof. Proof is easy, hence omitted.

Lemma 5.4. Let $\mathbf{H}$ be an LA-semihypergroup with left identity. Then for any $l_{r} \in$ $\mathbf{H},\left(l_{r} \underline{o} l_{r}\right) \underline{o} \mathbf{H}$ is an interior-hyperideal of $\mathbf{H}$.

Proof. Firstly, we will show that $\left(l_{r} \underline{\mathrm{o}} l_{r}\right) \underline{\mathrm{o}} \mathbf{H}$ is a sub-LA-semihypergroup of $\mathbf{H}$, for some $l_{r} \in \mathbf{H}$. So, we have

$$
\begin{aligned}
\left(\left(l_{r} \underline{\mathrm{o}} l_{r}\right) \underline{\mathrm{o}} \mathbf{H}\right) \underline{\mathrm{o}}\left(\left(l_{r} \underline{\mathrm{o}} l_{r}\right) \underline{\mathrm{o}} \mathbf{H}\right) & =\left(\left(\left(l_{r} \underline{\mathrm{o}} l_{r}\right) \underline{\mathrm{o}} \mathbf{H}\right) \underline{\mathrm{o}} \mathbf{H}\right) \underline{\mathrm{o}}\left(l_{r} \underline{\mathrm{o}} l_{r}\right) \\
& =\left((\mathbf{H} \underline{\mathrm{o}} \mathbf{H}) \underline{\mathrm{o}}\left(l_{r} \underline{\mathrm{o}} l_{r}\right)\right) \underline{\mathrm{o}}\left(l_{r} \underline{\mathrm{o}} l_{r}\right) \\
& \subseteq\left(\mathbf{H} \underline{\mathrm{o}}\left(l_{r} \underline{\mathrm{o}} l_{r}\right)\right) \underline{\mathrm{o}}\left(l_{r} \underline{\mathrm{o}} l_{r}\right) \\
& \subseteq(\mathbf{H} \underline{\mathrm{o}}(\mathbf{H} \underline{\mathrm{O}} \mathbf{H})) \underline{\mathrm{o}}\left(l_{r} \underline{\mathrm{o}} l_{r}\right) \\
& \subseteq(\mathbf{H} \underline{\mathrm{o}} \mathbf{H}) \underline{\mathrm{o}}\left(l_{r} \underline{\mathrm{o}} l_{r}\right) \\
& \subseteq\left(l_{r} \underline{\mathrm{o}} l_{r}\right) \underline{\mathrm{o}}(\mathbf{H} \underline{\mathbf{H}}) \\
& \subseteq\left(l_{r} \underline{\mathrm{o}} l_{r}\right) \underline{\mathbf{H}} .
\end{aligned}
$$

Also,

$$
\left(\mathbf{H} \underline{\mathrm{o}}\left(\left(l_{r} \underline{\mathrm{o}} l_{r}\right) \underline{\mathrm{o}} \mathbf{H}\right)\right) \underline{\mathrm{o}} \mathbf{H}=\left(\mathbf{H} \underline{\mathrm{o}}\left(\left(\mathbf{H} \underline{\mathrm{o}} l_{r}\right) \underline{\mathrm{o}} l_{r}\right)\right) \underline{\mathrm{o}} \mathbf{H}
$$




$$
\begin{aligned}
& =\left(\left(\mathbf{H} \underline{\mathrm{o}} l_{r}\right) \underline{\mathrm{o}}\left(\mathbf{H} \underline{\mathrm{o}} l_{r}\right)\right) \underline{\mathrm{o}} \mathbf{H} \\
& =\left((\mathbf{H} \underline{\mathrm{o}} \mathbf{H}) \underline{\mathrm{o}}\left(l_{r} \underline{\mathrm{o}} l_{r}\right)\right) \underline{\mathrm{o}} \mathbf{H} \\
& =\left(\left(l_{r} \underline{\mathrm{o}} l_{r}\right) \underline{\mathrm{o}}(\mathbf{H} \underline{\mathrm{o}} \mathbf{H})\right) \underline{\mathrm{o}} \mathbf{H} \\
& \subseteq\left(\left(l_{r} \underline{\mathrm{o}} l_{r}\right) \underline{\mathrm{o}} \mathbf{H}\right) \underline{\mathrm{o}} \mathbf{H} \\
& =(\mathbf{H} \underline{\mathrm{o}} \mathbf{H}) \underline{\mathrm{o}}\left(l_{r} \underline{\mathrm{o}} l_{r}\right) \\
& =\left(l_{r} \underline{\mathrm{o}} l_{r}\right) \underline{\mathrm{o}}(\mathbf{H} \underline{\mathrm{o}} \mathbf{H}) \\
& \subseteq\left(l_{r} \underline{\mathrm{o}} l_{r}\right) \underline{\mathrm{o}} \mathbf{H} .
\end{aligned}
$$

Hence, $\left(l_{r} \underline{\mathrm{o}} l_{r}\right) \underline{\mathrm{o}} \mathbf{H}$ is an interior-hyperideal of $\mathbf{H}$.

Theorem 5.1. Let $\mathbf{H}$ be an LA-semihypergroup with left identity, then the following statements are equivalent.

(1) $\mathbf{H}$ is left regular.

(2) $\mathcal{M} \subseteq \mathcal{M}^{2}$ and $\mathcal{M}$ is semiprime, where $\mathcal{M}$ is an interior-hyperideal of $\mathbf{H}$.

(3) $\mathcal{F}_{\mathbf{H}} \sqsubseteq \mathcal{F}_{\mathbf{H}} \hat{\diamond} \mathcal{F}_{\mathbf{H}}$ and $\mathcal{F}_{\mathbf{H}}$ is soft semiprime, where $\mathcal{F}_{\mathbf{H}}$ is an S.I. interior hypeerideal of $\mathbf{H}$ over $\mathcal{U}$.

Proof. (1) $\Rightarrow(3)$ Let $\mathbf{H}$ be a left regular LA-semihypergroup with left identity, thus for any $l_{r} \in \mathbf{H}$, there exists $x \in \mathbf{H}$ such that $l_{r} \in x \underline{\mathrm{o}}\left(l_{r} \underline{\mathrm{o}} l_{r}\right)$. Now we have

$$
\begin{aligned}
l_{r} & \in x \underline{\mathrm{o}}\left(l_{r} \underline{\mathrm{o}} l_{r}\right) \\
& =l_{r} \underline{\mathrm{o}}\left(x \underline{\mathrm{o}} l_{r}\right) \\
& \subseteq l_{r} \underline{\mathrm{o}}\left(x \underline{\mathrm{o}}\left(x \underline{\mathrm{o}}\left(l_{r} \underline{\mathrm{o}} l_{r}\right)\right)\right) \\
& \subseteq l_{r} \underline{\mathrm{o}}\left((e \underline{\mathrm{o}} x) \underline{\mathrm{o}}\left(l_{r} \underline{\mathrm{o}}\left(x \underline{\mathrm{o}} l_{r}\right)\right)\right) \\
& =l_{r} \underline{\mathrm{o}}\left(\left(\left(x \underline{\mathrm{o}} l_{r}\right) \underline{\mathrm{o}} l_{r}\right) \underline{\mathrm{o}}(x \underline{\mathrm{o}} e)\right) .
\end{aligned}
$$

Then, there exists $b \in x \underline{\mathrm{o}} l_{r}$ and $c \in x \underline{\mathrm{o}} e$ such that $l_{r} \in l_{r} \underline{\mathrm{o}}\left(\left(b \underline{\mathrm{o}} l_{r}\right) \underline{\mathrm{o}} c\right)$. Again, there exists $d \in\left(\left(b \underline{\mathrm{o}} l_{r}\right) \underline{\mathrm{o}} c\right)$ such that $l_{r} \in l_{r} \underline{\mathrm{o}} d$. So, $\left(l_{r}, d\right) \in \mathbb{H}_{l_{r}}$. Thus, we have

$$
\begin{aligned}
\left(\mathcal{F}_{\mathbf{H}} \hat{\diamond} \mathcal{F}_{\mathbf{H}}\right)\left(l_{r}\right) & =\bigcup_{(y, z) \in \mathbb{H}_{l_{r}}}\left\{\mathcal{F}_{\mathbf{H}}(y) \cap \mathcal{F}_{\mathbf{H}}(z)\right\} \\
& \supseteq \mathcal{F}_{\mathbf{H}}\left(l_{r}\right) \cap \mathcal{F}_{\mathbf{H}}(d) .
\end{aligned}
$$

As $\mathcal{F}_{\mathbf{H}}$ is an S.I. interior-hyperideal of $\mathbf{H}$ over $\mathcal{U}$, we have $\left.\bigcap_{\vartheta \in(x} \mathrm{O} y\right)$ o $z \mathcal{F}_{\mathbf{H}}(\vartheta) \supseteq \mathcal{F}_{\mathbf{H}}(y)$ for all $x, y, z \in \mathbf{H}$. Since $d \in\left(\left(b \underline{\mathrm{o}} l_{r}\right) \underline{\mathrm{o}} c\right)$ it would imply that $\mathcal{F}_{\mathbf{H}}(d) \supseteq \mathcal{F}_{\mathbf{H}}\left(l_{r}\right)$. Therefore from equation (5.2), we have

$$
\begin{aligned}
\left(\mathcal{F}_{\mathbf{H}} \hat{\diamond} \mathcal{F}_{\mathbf{H}}\right)\left(l_{r}\right) & \supseteq \mathcal{F}_{\mathbf{H}}\left(l_{r}\right) \cap \mathcal{F}_{\mathbf{H}}(d) \\
& \supseteq \mathcal{F}_{\mathbf{H}}\left(l_{r}\right) \cap \mathcal{F}_{\mathbf{H}}\left(l_{r}\right) \\
& =\mathcal{F}_{\mathbf{H}}\left(l_{r}\right) .
\end{aligned}
$$

Hence, $\mathcal{F}_{\mathbf{H}} \sqsubseteq \mathcal{F}_{\mathbf{H}} \hat{\diamond} \mathcal{F}_{\mathbf{H}}$. 
Now, it remains to show that $\mathcal{F}_{\mathbf{H}}$ is soft semiprime. Let $l_{r} \in \mathbf{H}$, then there exists $x \in \mathbf{H}$ such that $l_{r} \in x \underline{\mathrm{o}}\left(l_{r} \underline{\mathrm{o}} l_{r}\right)$. Therefore, we have

$$
\begin{aligned}
l_{r} & \in x \underline{\mathrm{o}}\left(l_{r} \underline{\mathrm{o}} l_{r}\right) \\
& \subseteq(e \underline{\mathrm{o}} x) \underline{\mathrm{o}}\left(l_{r} \underline{\mathrm{o}} l_{r}\right) \\
& =\left(l_{r} \underline{\mathrm{o}} l_{r}\right) \underline{\mathrm{o}}(x \underline{\mathrm{o}} e) \\
& \subseteq\left(l_{r} \underline{\mathrm{o}}\left(x \underline{\mathrm{o}}\left(l_{r} \underline{\mathrm{o}} l_{r}\right)\right)\right) \underline{\mathrm{o}}(x \underline{\mathrm{o}} e) \\
& =\left(x \underline{\mathrm{o}}\left(l_{r} \underline{\mathrm{o}}\left(l_{r} \underline{\mathrm{o}} l_{r}\right)\right)\right) \underline{\mathrm{o}}(x \underline{\mathrm{o}} e) \\
& \subseteq\left(x \underline{\mathrm{o}}\left(\left(e \underline{\mathrm{o}} l_{r}\right) \underline{\mathrm{o}}\left(l_{r} \underline{\mathrm{O}} l_{r}\right)\right)\right) \underline{\mathrm{o}}(x \underline{\mathrm{o}} e) \\
& =\left(x \underline{\mathrm{o}}\left(\left(l_{r} \underline{\mathrm{o}} l_{r}\right) \underline{\mathrm{o}}\left(l_{r} \underline{\mathrm{o}} e\right)\right)\right) \underline{\mathrm{o}}(x \underline{\mathrm{o}} e) \\
& =\left(\left(l_{r} \underline{\mathrm{o}} l_{r}\right) \underline{\mathrm{o}}\left(x \underline{\mathrm{o}}\left(l_{r} \underline{\mathrm{o}} e\right)\right)\right) \underline{\mathrm{o}}(x \underline{\mathrm{o}} e) \\
& =\left(\left(\left(l_{r} \underline{\mathrm{o}} e\right) \underline{\mathrm{o}} x\right) \underline{\mathrm{o}}\left(l_{r} \underline{\mathrm{o}} l_{r}\right)\right) \underline{\mathrm{o}}(x \underline{\mathrm{o}} e) .
\end{aligned}
$$

Then, we have

$$
\begin{aligned}
& \mathcal{F}_{\mathbf{H}}\left(l_{r}\right) \supseteq \bigcap_{\vartheta \in\left(\left(\left(l_{r} \underline{\mathrm{O}} e\right) \underline{\mathrm{O}} x\right) \underline{\mathrm{O}}\left(l_{r} \underline{\mathrm{O}} l_{r}\right)\right) \underline{\mathrm{O}}(x \underline{\mathrm{O}} e)} \mathcal{F}_{\mathbf{H}}(\vartheta) \\
& \supseteq \bigcap_{\vartheta \in\left(l_{r} \underline{\mathrm{O}} l_{r}\right)} \mathcal{F}_{\mathbf{H}}(\vartheta) \text {. }
\end{aligned}
$$

It implies $\mathcal{F}_{\mathbf{H}}$ is soft semiprime.

$(3) \Rightarrow(2)$ Assume that $\mathcal{M}$ is an interior-hyperideal of $\mathbf{H}$, then by Theorem $4.2 \mathcal{H}_{\mathcal{M}}$ will be an S.I. interior-hyperideal of $\mathbf{H}$ over $\mathcal{U}$. Let $m \in \mathcal{M}$, then we have $\mathcal{H}_{\mathcal{M}}(m)=\mathcal{U}$. Now

$$
\begin{aligned}
\mathcal{U} & =\mathcal{H}_{\mathcal{M}}(m) \\
& \subseteq\left(\mathcal{H}_{\mathcal{M}} \hat{\diamond} \mathcal{H}_{\mathcal{M}}\right)(m) \\
& =\mathcal{H}_{\left(\mathcal{M} \underline{\mathrm{o}} \mathcal{M}^{\prime}\right)}(m) .
\end{aligned}
$$

It would yield $m \in \mathcal{M} \underline{\mathrm{o}} \mathcal{\mathcal { M }}$. Therefore, $\mathcal{M} \subseteq \mathcal{M} \underline{\underline{o}} \mathcal{M}$. Now, let $m \underline{\mathrm{o}} m \subseteq \mathcal{M}$ for some $m \in \mathcal{M}$, then $\bigcap_{\vartheta \in(m \underline{\mathrm{O}} m)} \mathcal{H}_{\mathcal{M}}(\vartheta)=\mathcal{U}$. As $\mathcal{H}_{\mathcal{M}}$ is soft semiprime, thus we have

$$
\begin{aligned}
\mathcal{H}_{\mathcal{M}}(m) & \supseteq \bigcap_{\vartheta \in(m \underline{\mathrm{O}} m)} \mathcal{H}_{\mathcal{M}}(\vartheta) \\
& =\mathcal{U} .
\end{aligned}
$$

It follows that $m \in \mathcal{M}$. Hence, $\mathcal{M}$ is semiprime.

$(2) \Rightarrow(1)$ By Lemma $5.4,\left(l_{r} \underline{\mathrm{o}} l_{r}\right) \underline{\mathrm{o}} \mathbf{H}$ is an interior-hyperideal of $\mathbf{H}$. Now, $l_{r} \underline{\mathrm{o}} l_{r} \subseteq$ $\left(l_{r} \underline{\mathrm{o}} l_{r}\right) \underline{\mathrm{O}} \mathbf{H}$ for some $l_{r} \in \mathbf{H}$, then by assumption $\left(l_{r} \underline{\mathrm{o}} l_{r}\right) \underline{\mathrm{o}} \mathbf{H}$ will be semiprime. Thus, it would imply that $l_{r} \in\left(l_{r} \underline{\mathrm{o}} l_{r}\right) \underline{\mathrm{o}} \mathbf{H}$. Therefore, we have

$$
\begin{aligned}
l_{r} & \in\left(l_{r} \underline{\mathrm{o}} l_{r}\right) \underline{\mathrm{o}} \mathbf{H} \\
& \subseteq\left(\left(l_{r} \underline{\mathrm{o}} l_{r}\right) \underline{\mathrm{o}} \mathbf{H}\right) \underline{\mathrm{o}}\left(\left(l_{r} \underline{\mathrm{o}} l_{r}\right) \underline{\mathrm{o}} \mathbf{H}\right) \\
& =\left(\mathbf{H} \underline{\mathrm{o}}\left(l_{r} \underline{\mathrm{o}} l_{r}\right)\right) \underline{\mathrm{o}}\left(\mathbf{H} \underline{\mathrm{o}}\left(l_{r} \underline{\mathrm{o}} l_{r}\right)\right)
\end{aligned}
$$




$$
\begin{aligned}
& \subseteq\left(\mathbf{H}_{\underline{\mathrm{o}}} \mathbf{H}\right) \underline{\mathrm{o}}\left((e \underline{\mathrm{o}} \mathbf{H}) \underline{\mathrm{o}}\left(l_{r} \underline{\mathrm{o}} l_{r}\right)\right) \\
& \subseteq \underline{\mathbf{H}} \underline{\mathrm{o}}\left(\left(l_{r} \underline{\mathrm{o}} l_{r}\right) \underline{\mathrm{o}}(\mathbf{H} \underline{\mathrm{o}} e)\right) \\
& \subseteq\left(l_{r} \underline{\mathrm{o}} l_{r}\right) \underline{\mathrm{o}}(\mathbf{H} \underline{\mathrm{o}}(\mathbf{H} \underline{\mathrm{o}} \mathbf{H})) \\
& \subseteq\left(l_{r} \underline{\mathrm{o}} l_{r}\right) \underline{\mathrm{o}}(\mathbf{H} \underline{\mathrm{o}} \mathbf{H}) \\
& =\left(\mathbf{H}^{\mathrm{o}} \mathbf{H}\right) \underline{\mathrm{o}}\left(l_{r} \underline{\mathrm{o}} l_{r}\right) \\
& \subseteq \mathbf{H} \underline{\mathrm{o}}\left(l_{r} \underline{\mathrm{o}} l_{r}\right) .
\end{aligned}
$$

Hence, $\mathbf{H}$ is left regular.

Theorem 5.2. If $\mathbf{H}$ is a left regular LA-semihypergroup with left identity, then every S.I. interior-hyperideal of $\mathbf{H}$ over $\mathcal{U}$ is idempotent.

Proof. Let $\mathbf{H}$ be an LA-semihypergroup with left identity and let $l_{r} \in \mathbf{H}$. As $\mathbf{H}$ is left regular, thus for any $l_{r} \in \mathbf{H}$, there exists $x \in \mathbf{H}$ such that

$$
\begin{aligned}
l_{r} & \in x \underline{\mathrm{o}}\left(l_{r} \underline{\mathrm{o}} l_{r}\right) \\
& =l_{r} \underline{\mathrm{o}}\left(x \underline{\mathrm{o}} l_{r}\right) \\
& \subseteq\left(x \underline{\mathrm{o}}\left(l_{r} \underline{\mathrm{o}} l_{r}\right)\right) \underline{\mathrm{o}}\left(x \underline{\mathrm{o}} l_{r}\right) \\
& =\left(l_{r} \underline{\mathrm{o}} x\right) \underline{\mathrm{o}}\left(\left(l_{r} \underline{\mathrm{o}} l_{r}\right) \underline{\mathrm{o}} x\right) \\
& =\left(\left(\left(l_{r} \underline{\mathrm{o}} l_{r}\right) \underline{\mathrm{o}} x\right) \underline{\mathrm{o}} x\right) \underline{\mathrm{o}} l_{r} \\
& =\left((x \underline{\mathrm{o}} x) \underline{\mathrm{o}}\left(l_{r} \underline{\mathrm{o}} l_{r}\right)\right) \underline{\mathrm{o}} l_{r} \\
& =\left(\left(l_{r} \underline{\mathrm{o}} l_{r}\right) \underline{\mathrm{o}}(x \underline{\mathrm{o}} x)\right) \underline{\mathrm{o}} l_{r} .
\end{aligned}
$$

Then, there exists $b \in\left(l_{r} \underline{\mathrm{O}} l_{r}\right) \underline{\mathrm{O}}(x \underline{\mathrm{O}} x)$ such that $l_{r} \in b \underline{\mathrm{o}} l_{r}$. Therefore $\left(b, l_{r}\right) \in$ $\mathbb{H}_{l_{r}}$. Suppose $\mathcal{F}_{\mathbf{H}}$ is an S.I. interior-hyperideal of $\mathbf{H}$ over $\mathcal{U}$, then by Lemma 5.2, $\mathcal{H}_{\mathbf{H}} \hat{\diamond} \mathcal{F}_{\mathbf{H}}=\mathcal{F}_{\mathbf{H}}$. Thus, $\mathcal{F}_{\mathbf{H}} \hat{\diamond} \mathcal{F}_{\mathbf{H}} \sqsubseteq \mathcal{H}_{\mathbf{H}} \hat{\diamond} \mathcal{F}_{\mathbf{H}}=\mathcal{F}_{\mathbf{H}}$. Now, it remain to prove that $\mathcal{F}_{\mathbf{H}} \hat{\diamond} \mathcal{F}_{\mathbf{H}} \sqsupseteq \mathcal{F}_{\mathbf{H}}$. For this, we have

$$
\begin{aligned}
\left(\mathcal{F}_{\mathbf{H}} \hat{\diamond} \mathcal{F}_{\mathbf{H}}\right)\left(l_{r}\right) & =\bigcup_{(y, z) \in \mathbb{H}_{l_{r}}}\left\{\mathcal{F}_{\mathbf{H}}(y) \cap \mathcal{F}_{\mathbf{H}}(z)\right\} \\
& \supseteq \mathcal{F}_{\mathbf{H}}(b) \cap \mathcal{F}_{\mathbf{H}}\left(l_{r}\right) .
\end{aligned}
$$

As $\mathcal{F}_{\mathbf{H}}$ is an S.I. interior-hyperideal of $\mathbf{H}$ over $\mathcal{U}$, we have $\left.\bigcap_{\vartheta \in(x} \underline{\mathrm{O}} y\right) \underline{\mathrm{o}} z \mathcal{F}_{\mathbf{H}}(\vartheta) \supseteq \mathcal{F}_{\mathbf{H}}(y)$. Since $b \in\left(l_{r} \underline{\mathrm{o}} l_{r}\right) \underline{\mathrm{o}}(x \underline{\mathrm{o}} x)$, it would imply that $\mathcal{F}_{\mathbf{H}}(b) \supseteq \mathcal{F}_{\mathbf{H}}\left(l_{r}\right)$. Hence, from (5.3), we have

$$
\begin{aligned}
\left(\mathcal{F}_{\mathbf{H}} \hat{\diamond} \mathcal{F}_{\mathbf{H}}\right)\left(l_{r}\right) & \supseteq \mathcal{F}_{\mathbf{H}}(b) \cap \mathcal{F}_{\mathbf{H}}\left(l_{r}\right) \\
& \supseteq \mathcal{F}_{\mathbf{H}}\left(l_{r}\right) \cap \mathcal{F}_{\mathbf{H}}\left(l_{r}\right) \\
& =\mathcal{F}_{\mathbf{H}}\left(l_{r}\right) .
\end{aligned}
$$

This shows that every S.I. interior-hyperideal of $\mathbf{H}$ is idempotent.

Theorem 5.3. If $\mathbf{H}$ is an LA-semihypergroup with left identity, then the following statements are equivalent. 
(1) $\mathbf{H}$ is left regular.

(2) Every S.I. interior-hyperideal of $\mathbf{H}$ over $\mathcal{U}$ is soft semiprime and idempotent.

Proof. (1) $\Rightarrow(2)$ Let $\mathbf{H}$ be a left regular LA-semihypergroup with left identity and let $\mathcal{F}_{\mathbf{H}}$ be an S.I. interior-hyperideal of $\mathbf{H}$ over $\mathcal{U}$, then by Theorem $5.2, \mathcal{F}_{\mathbf{H}}$ will be idempotent. Thus, it is only remains to show that $\mathcal{F}_{\mathbf{H}}$ is soft semiprime. As $\mathbf{H}$ is left regular, thus for any $l_{r} \in \mathbf{H}$ there exists $x \in \mathbf{H}$ such that

$$
\begin{aligned}
l_{r} & \in x \underline{\mathrm{o}}\left(l_{r} \underline{\mathrm{o}} l_{r}\right) \\
& \subseteq(e \underline{\mathrm{o}} x) \underline{\mathrm{o}}\left(l_{r} \underline{\mathrm{o}} l_{r}\right) \\
& =\left(l_{r} \underline{\mathrm{o}} l_{r}\right) \underline{\mathrm{o}}(x \underline{\mathrm{o}} e) \\
& \subseteq\left(l_{r} \underline{\mathrm{o}}\left(e \underline{\mathrm{o}} l_{r}\right)\right) \underline{\mathrm{o}}(x \underline{\mathrm{o}} e) \\
& =\left(e \underline{\mathrm{o}}\left(l_{r} \underline{\mathrm{o}} l_{r}\right)\right) \underline{\mathrm{o}}(x \underline{\mathrm{o}} e) .
\end{aligned}
$$

Then, we have

$$
\begin{aligned}
& \mathcal{F}_{\mathbf{H}}\left(l_{r}\right) \supseteq \bigcap_{\vartheta \in\left(\left(e \underline{\mathrm{O}}\left(l_{r} \underline{\mathrm{O}} l_{r}\right)\right) \underline{\mathrm{O}}(x \underline{\mathrm{O}} e)\right)} \mathcal{F}_{\mathbf{H}}(\vartheta) \\
& \supseteq \bigcap_{\vartheta \in l_{r}} \underline{\mathrm{O}} l_{r} \\
& \mathcal{F}_{\mathbf{H}}(\vartheta) . \text { As } \mathcal{F}_{\mathbf{H}} \text { is an S.I. interior hyperideal of } \mathbf{H} .
\end{aligned}
$$

Hence, $\mathcal{F}_{\mathbf{H}}$ is soft semiprime.

$(2) \Rightarrow(1)$ Suppose that every S.I. interior-hyperideal of $\mathbf{H}$ over $\mathcal{U}$ with left identity is idempotent and soft semiprime. By Lemma 5.4, $\left(l_{r} \underline{\mathrm{o}} l_{r}\right) \underline{\mathrm{o}} \mathbf{H}$ is an interior-hyperideal of $\mathbf{H}$. Therefore, by Theorem 4.2, characteristic soft function $\mathcal{H}_{\left(l_{r} \underline{\mathrm{O}} l_{r}\right)} \underline{\mathrm{O}} \mathbf{H}$ will be S.I.

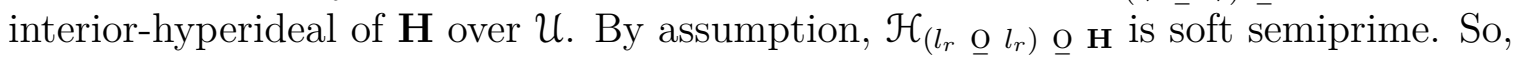
by Lemma 5.3, $\left(l_{r} \underline{\mathrm{o}} l_{r}\right) \underline{\mathrm{o}} \mathbf{H}$ will be semiprime. Thus, for any $l_{r} \in \mathbf{H}$, we have

$$
\begin{aligned}
\left(l_{r} \underline{\mathrm{o}} l_{r}\right) & \subseteq\left(e \underline{\mathrm{o}} l_{r}\right) \underline{\mathrm{o}} l_{r} \\
& =\left(l_{r} \underline{\mathrm{o}} l_{r}\right) \underline{\mathrm{o}} e \\
& \subseteq\left(l_{r} \underline{\mathrm{o}} l_{r}\right) \underline{\mathrm{o}} H .
\end{aligned}
$$

This yield $l_{r} \in\left(l_{r} \underline{\mathrm{o}} l_{r}\right) \underline{\mathrm{o}} \mathbf{H}$. Therefore, we have

$$
\begin{aligned}
l_{r} & \in\left(l_{r} \underline{\mathrm{o}} l_{r}\right) \underline{\mathrm{o}} \mathbf{H} \\
& =\left(l_{r} \underline{\mathrm{o}} l_{r}\right) \underline{\mathrm{o}}(\mathbf{H} \underline{\mathrm{o}} \mathbf{H}) \\
& =(\mathbf{H} \underline{\mathrm{o}} \mathbf{H}) \underline{\mathrm{o}}\left(l_{r} \underline{\mathrm{o}} l_{r}\right) \\
& \subseteq \mathbf{H} \underline{\mathrm{o}}\left(l_{r} \underline{\mathrm{o}} l_{r}\right) .
\end{aligned}
$$

Hence, $\mathbf{H}$ is left regular.

Theorem 5.4. Let $\mathbf{H}$ be a left regular LA-semihypergroup with left identity, then $\left(\mathcal{H}_{\mathbf{H}} \hat{\diamond} \mathcal{F}_{\mathbf{H}}\right) \hat{\diamond}\left(\mathcal{H}_{\mathbf{H}} \hat{\diamond} \mathcal{F}_{\mathbf{H}}\right)=\mathcal{F}_{\mathbf{H}}$, for every S.I. interior-hyperideal $\mathcal{F}_{\mathbf{H}}$ of $\mathbf{H}$ over $\mathcal{U}$.

Proof. Assume that $\mathbf{H}$ is a left regular LA-semihypergroup with left identity. Let $\mathcal{F}_{\mathbf{H}}$ be any S.I. interior-hyperideal of $\mathbf{H}$ over $\mathcal{U}$, then by Theorem $5.3, \mathcal{F}_{\mathbf{H}}$ will be soft 
semiprime and idempotent. Also, by Lemma 5.2, $\mathcal{H}_{\mathbf{H}} \hat{\diamond} \mathcal{F}_{\mathbf{H}}=\mathcal{F}_{\mathbf{H}}$. Thus, we have

$$
\begin{aligned}
\left(\mathcal{H}_{\mathbf{H}} \hat{\diamond} \mathcal{F}_{\mathbf{H}}\right) \hat{\diamond}\left(\mathcal{H}_{\mathbf{H}} \hat{\diamond} \mathcal{F}_{\mathbf{H}}\right) & =\mathcal{F}_{\mathbf{H}} \hat{\diamond} \mathcal{F}_{\mathbf{H}} \\
& =\mathcal{F}_{\mathbf{H}} .
\end{aligned}
$$

Hence, $\left(\mathcal{H}_{\mathbf{H}} \hat{\diamond} \mathcal{F}_{\mathbf{H}}\right) \hat{\diamond}\left(\mathcal{H}_{\mathbf{H}} \hat{\diamond} \mathcal{F}_{\mathbf{H}}\right)=\mathcal{F}_{\mathbf{H}}$.

Theorem 5.5. Let $\mathbf{H}$ be an LA-semihypergroup with left identity, then the following statements are equivalent.

(1) $\mathbf{H}$ is left regular.

(2) Every S.I. interior-hyperideal of $\mathbf{H}$ over $\mathcal{U}$ is soft semiprime.

(3) $\mathcal{F}_{\mathbf{H}}(h)=\bigcap_{\vartheta \in l_{r}} \underline{o} l_{r} \mathcal{F}_{\mathbf{H}}(\vartheta)$, for every S.I. interior-hyperideal $\mathcal{F}_{\mathbf{H}}$ of $\mathbf{H}$ over $\mathcal{U}$, for all $l_{r} \in \mathbf{H}$.

Proof. (1) $\Rightarrow(2)$ Suppose that $\mathcal{F}_{\mathbf{H}}$ is an S.I. interior-hyperideal of $\mathbf{H}$ over $\mathcal{U}$. As $\mathbf{H}$ is left regular, thus for any $l_{r} \in \mathbf{H}$, there exists $x \in \mathbf{H}$ such that $l_{r} \in x \underline{\mathrm{o}}\left(l_{r} \underline{\mathrm{o}} l_{r}\right)$. Now, we have

$$
\begin{aligned}
l_{r} & \in x \underline{\mathrm{o}}\left(l_{r} \underline{\mathrm{o}} l_{r}\right) \\
& \subseteq x \underline{\mathrm{o}}\left(\left(x \underline{\mathrm{o}}\left(l_{r} \underline{\mathrm{o}} l_{r}\right)\right) \underline{\mathrm{o}} l_{r}\right) \\
& =\left(x \underline{\mathrm{o}}\left(l_{r} \underline{\mathrm{o}} l_{r}\right)\right) \underline{\mathrm{o}}\left(x \underline{\mathrm{o}} l_{r}\right) .
\end{aligned}
$$

As $\mathcal{F}_{\mathbf{H}}$ is an S.I. interior-hyperideal of $\mathbf{H}$ over $\mathcal{U}$, we have $\left.\bigcap_{\vartheta \in(x} \underline{\mathrm{o}} y\right) \underline{\mathrm{o}} z \mathcal{F}_{\mathbf{H}}(\vartheta) \supseteq \mathcal{F}_{\mathbf{H}}(y)$ for all $x, y, z \in \mathbf{H}$. Since $l_{r} \in\left(x \underline{\mathrm{o}}\left(l_{r} \underline{\mathrm{o}} l_{r}\right)\right) \underline{\mathrm{o}}\left(x \underline{\mathrm{o}} l_{r}\right)$, it would imply that $\left.\left.\mathcal{F}_{\mathbf{H}}\left(l_{r}\right) \supseteq \bigcap_{\vartheta \in(x} \underline{\mathrm{O}}\left(l_{r} \underline{\mathrm{O}} l_{r}\right)\right) \underline{\mathrm{o}}\left(x \underline{\mathrm{O}} l_{r}\right) \mathcal{F}_{\mathbf{H}}(\vartheta) \supseteq \bigcap_{\vartheta \in\left(l_{r}\right.} \underline{\mathrm{O}} l_{r}\right) \mathcal{F}_{\mathbf{H}}(\vartheta)$. Therefore, $\mathcal{F}_{\mathbf{H}}$ is soft semiprime.

$(2) \Rightarrow(3)$ Here, we only need to show that $\bigcap_{\vartheta \in l_{r}} \underline{\mathrm{o} l_{r}} \mathcal{F}_{\mathbf{H}}(\vartheta) \supseteq \mathcal{F}_{\mathbf{H}}\left(l_{r}\right)$. For this, we have

$$
\begin{aligned}
l_{r} \underline{\mathrm{o}} l_{r} & \subseteq l_{r} \underline{\mathrm{o}}\left(x \underline{\mathrm{o}}\left(l_{r} \underline{\mathrm{o}} l_{r}\right)\right) \\
& =l_{r} \underline{\mathrm{o}}\left(l_{r} \underline{\mathrm{o}}\left(x \underline{\mathrm{o}} l_{r}\right)\right) \\
& \subseteq\left(e \underline{\mathrm{o}} l_{r}\right) \underline{\mathrm{o}}\left(l_{r} \underline{\mathrm{o}}\left(x \underline{\mathrm{o}} l_{r}\right)\right) \\
& =\left(\left(x \underline{\mathrm{o}} l_{r}\right) \underline{\mathrm{o}} l_{r}\right) \underline{\mathrm{o}}\left(l_{r} \underline{\mathrm{o}} e\right) .
\end{aligned}
$$

Then, we have

$$
\begin{aligned}
& \bigcap_{\vartheta \in\left(l_{r} \underline{\mathrm{O}} l_{r}\right)} \mathcal{F}_{\mathbf{H}}(\vartheta) \supseteq \bigcap_{\vartheta \in\left(\left(x \underline{\mathrm{O}} l_{r}\right) \underline{\mathrm{O}} l_{r}\right) \underline{\mathrm{O}}\left(l_{r} \underline{\mathrm{O}} e\right)} \mathcal{F}_{\mathbf{H}}(\vartheta) \\
& \supseteq \mathcal{F}_{\mathbf{H}}\left(l_{r}\right)
\end{aligned}
$$

(as $\mathcal{F}_{\mathbf{H}}$ is an S.I. interior hyperideal).

It follows that $\mathcal{F}_{\mathbf{H}}\left(l_{r}\right)=\bigcap_{\vartheta \in l_{r}} \underline{\mathrm{o}} l_{r} \mathcal{F}_{\mathbf{H}}(\vartheta)$.

$(3) \Rightarrow(1)$ By Lemma 5.4, $\left(l_{r} \underline{\mathrm{o}} l_{r}\right) \underline{\mathrm{o}} \mathbf{H}$ is an interior-hyperideal of $\mathbf{H}$. Now

$$
\left(l_{r} \underline{\mathrm{o}} l_{r}\right) \subseteq\left(e \underline{\mathrm{o}} l_{r}\right) \underline{\mathrm{o}} l_{r}
$$




$$
\begin{aligned}
& =\left(l_{r} \underline{\mathrm{O}} l_{r}\right) \underline{\mathrm{o}} e \\
& \subseteq\left(l_{r} \underline{\mathrm{o}} l_{r}\right) \underline{\mathbf{o}} \mathbf{H} .
\end{aligned}
$$

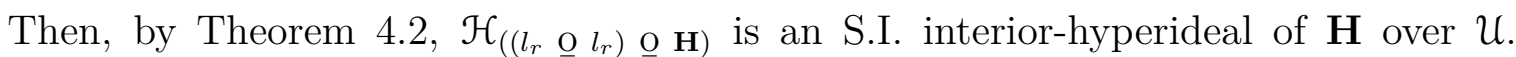
Now, $\left(l_{r} \underline{\mathrm{o}} l_{r}\right) \subseteq\left(l_{r} \underline{\mathrm{o}} l_{r}\right) \underline{\mathrm{o}} \mathbf{H}$, it would imply $\bigcap_{\vartheta \in l_{r}} \mathrm{O} l_{r} \mathcal{H}_{\left(\left(l_{r} \mathrm{O} l_{r}\right) \mathrm{O} \mathbf{H}\right)}(\vartheta)=\mathcal{U}$. By assumption, $\mathcal{H}_{\left(\left(l_{r} \underline{\mathrm{O}} l_{r}\right) \underline{\mathrm{O}} \mathbf{H}\right)}\left(l_{r}\right)=\bigcap_{\vartheta \in l_{r}} \underline{\mathrm{O}} l_{r} \mathcal{H}_{\left(\left(l_{r} \underline{\mathrm{O}} l_{r}\right) \underline{\mathrm{O}} \mathbf{H}\right)}(\vartheta)=\mathcal{U}$. This yield $l_{r} \in$ $\left(l_{r} \underline{\mathrm{o}} l_{r}\right) \underline{\mathrm{o}} \mathbf{H}$. Therefore,

$$
\begin{aligned}
l_{r} & \in\left(l_{r} \underline{\mathrm{o}} l_{r}\right) \underline{\mathrm{o}} \mathbf{H} \\
& =\left(l_{r} \underline{\mathrm{o}} l_{r}\right) \underline{\mathrm{o}}(\mathbf{H} \underline{\mathrm{o}} \mathbf{H}) \\
& =(\mathbf{H} \underline{\mathrm{o}} \mathbf{H}) \underline{\mathrm{o}}\left(l_{r} \underline{\mathrm{o}} l_{r}\right) \\
& \subseteq \mathbf{H} \underline{\mathrm{o}}\left(l_{r} \underline{\mathrm{o}} l_{r}\right) .
\end{aligned}
$$

Hence, $\mathbf{H}$ is left regular.

Theorem 5.6. Let $\mathbf{H}$ be a left regular LA-semihypergroup with left identity, then the following statements are equivalent:

(1) $\mathcal{F}_{\mathbf{H}}$ is an S.I. interior-hyperideal of $\mathbf{H}$ over $\boldsymbol{U}$;

(2) $\mathcal{F}_{\mathbf{H}}$ is an S.I. bi-hyperideal of $\mathbf{H}$ over $\mathcal{U}$.

Proof. (1) $\Rightarrow(2)$ Let $\mathbf{H}$ be a left regular LA-semihypergroup with left identity, thus for $a, b \in \mathbf{H}$, there exists $a^{\prime}, b^{\prime} \in \mathbf{H}$ such that $a \in a^{\prime} \underline{\mathrm{o}}(a \underline{\mathrm{o}} a)$ and $b \in b^{\prime} \underline{\mathrm{o}}(b \underline{\mathrm{o}} b)$. Suppose that $\mathcal{F}_{\mathbf{H}}$ is an S.I. interior-hyperideal of $\mathbf{H}$ over $\mathcal{U}$. Then, we have

$$
\begin{aligned}
\bigcap_{\vartheta \in\left(\left(a \underline{\mathrm{O}} l_{r}\right) \underline{\mathrm{O}} b\right)} \mathcal{F}_{\mathbf{H}}(\vartheta) & \supseteq \bigcap_{\vartheta \in\left(\left(a^{\prime} \underline{\mathrm{O}}(a \underline{\mathrm{O}} a)\right) \underline{\mathrm{O}} l_{r}\right) \underline{\mathrm{O}} b} \mathcal{F}_{\mathbf{H}}(\vartheta) \\
& =\bigcap_{\vartheta \in\left(\left(a \underline{\mathrm{O}}\left(a^{\prime} \underline{\mathrm{O}} a\right)\right) \underline{\mathrm{O}} l_{r}\right) \underline{\mathrm{O}} b} \mathcal{F}_{\mathbf{H}}(\vartheta) \\
& =\bigcap_{\vartheta \in\left(\left(l_{r} \underline{\mathrm{O}}\left(a^{\prime} \underline{\mathrm{O}} a\right)\right) \underline{\mathrm{O}} a\right) \underline{\mathrm{O}} b} \mathcal{F}_{\mathbf{H}}(\vartheta) \\
& \supseteq \mathcal{F}_{\mathbf{H}}(a) .
\end{aligned}
$$

Also, we have

$$
\begin{aligned}
\bigcap_{\vartheta \in\left(\left(a \underline{\mathrm{O}} l_{r}\right) \underline{\mathrm{O}} b\right)} \mathcal{F}_{\mathbf{H}}(\vartheta) & \supseteq \bigcap_{\vartheta \in\left(\left(a \underline{\mathrm{O}} l_{r}\right) \underline{\mathrm{O}}\left(b^{\prime} \underline{\mathrm{O}}(b \underline{\mathrm{O}} b)\right)\right)} \mathcal{F}_{\mathbf{H}}(\vartheta) \\
& =\bigcap_{\vartheta \in\left(\left(a \underline{\mathrm{O}} l_{r}\right) \underline{\mathrm{O}}\left(b \underline{\mathrm{O}}\left(b^{\prime} \underline{\mathrm{O}} b\right)\right)\right)} \mathcal{F}_{\mathbf{H}}(\vartheta) \\
& =\bigcap_{\vartheta \in\left((a \underline{\mathrm{O}} b) \underline{\mathrm{O}}\left(l_{r} \underline{\mathrm{O}}\left(b^{\prime} \underline{\mathrm{O}} b\right)\right)\right)} \mathcal{F}_{\mathbf{H}}(\vartheta) \\
& \supseteq \mathcal{F}_{\mathbf{H}}(b) .
\end{aligned}
$$

This show that $\bigcap_{\vartheta \in\left(\left(a \underline{\mathrm{O}} l_{r}\right) \underline{\mathrm{O}} b\right)} \mathcal{F}_{\mathbf{H}}(\vartheta) \supseteq \mathcal{F}_{\mathbf{H}}(a) \cap \mathcal{F}_{\mathbf{H}}(b)$. Hence, $\mathcal{F}_{\mathbf{H}}$ is an S.I. bihyperideal of $\mathbf{H}$ over $\mathcal{U}$. 
$(2) \Rightarrow(1)$ Suppose that $\mathbf{H}$ is a left regular LA-semihypergroup with left identity ' $e^{\prime}$ and $\mathcal{F}_{\mathbf{H}}$ an S.I. bi-hyperideal of $\mathbf{H}$ over $\mathcal{U}$. Let $l_{r} \in \mathbf{H}$, then there exists $l_{r}^{\prime} \in \mathbf{H}$ such that $l_{r} \in l_{r}^{\prime} \underline{\mathrm{o}}\left(l_{r} \underline{\mathrm{o}} l_{r}\right)$. Then for any $x, y \in \mathbf{H}$, we have

$$
\begin{aligned}
& \bigcap_{\vartheta \in\left(\left(x \underline{\mathrm{O}} l_{r}\right) \underline{\mathrm{O}} y\right)} \mathcal{F}_{\mathbf{H}}(\vartheta) \supseteq \bigcap_{\vartheta \in\left(\left(x \underline{\mathrm{O}} l_{r}\right) \underline{\mathrm{O}}(e \underline{\mathrm{O}} y)\right)} \mathcal{F}_{\mathbf{H}}(\vartheta) \\
& =\bigcap_{\vartheta \in\left((y \underline{\mathrm{O}} e) \underline{\mathrm{O}}\left(l_{r} \underline{\mathrm{O}} x\right)\right)} \mathcal{F}_{\mathbf{H}}(\vartheta) \\
& =\bigcap_{\vartheta \in\left(l_{r} \underline{\mathrm{O}}((y \underline{\mathrm{O}} e) \underline{\mathrm{O}} x)\right.} \mathcal{F}_{\mathbf{H}}(\vartheta) \\
& \supseteq \bigcap_{\vartheta \in\left(\left(l_{r}^{\prime} \underline{\mathrm{O}}\left(l_{r} \underline{\mathrm{O}} l_{r}\right)\right) \underline{\mathrm{O}}((y \underline{\mathrm{O}} e) \underline{\mathrm{O}} x)\right)} \mathcal{F}_{\mathbf{H}}(\vartheta) \\
& =\bigcap_{\vartheta \in\left(\left(l_{r} \underline{\mathrm{O}}\left(l_{r}^{\prime} \underline{\mathrm{O}} l_{r}\right)\right) \underline{\mathrm{O}}\left(\left(\begin{array}{llll}
y & \mathrm{O} & e
\end{array}\right) \underline{\mathrm{O}} x\right)\right.} \mathcal{F}_{\mathbf{H}}(\vartheta)
\end{aligned}
$$

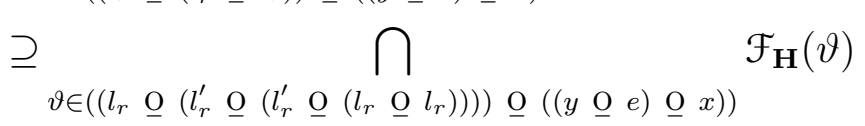

$$
\begin{aligned}
& =\bigcap_{\vartheta \in\left(\left(((y \underline{\mathrm{O}} e) \underline{\mathrm{O}} x) \underline{\mathrm{O}}\left(l_{r}^{\prime} \underline{\mathrm{O}}\left(l_{r}^{\prime} \underline{\mathrm{O}}\left(l_{r} \underline{\mathrm{O}} l_{r}\right)\right)\right)\right) \underline{\mathrm{O}} l_{r}\right)} \mathcal{F}_{\mathbf{H}}(\vartheta) \\
& =\bigcap_{\vartheta \in\left(\left(((y \underline{\mathrm{O}} e) \underline{\mathrm{O}} x) \underline{\mathrm{O}}\left(l_{r}^{\prime} \underline{\mathrm{O}}\left(l_{r} \underline{\mathrm{O}}\left(l_{r}^{\prime} \underline{\mathrm{O}} l_{r}\right)\right)\right)\right) \underline{\mathrm{O}} l_{r}\right)} \mathcal{F}_{\mathbf{H}}(\vartheta) \\
& =\bigcap_{\vartheta \in\left(\left(((y \underline{\mathrm{O}} e) \underline{\mathrm{O}} x) \underline{\mathrm{O}}\left(l_{r} \underline{\mathrm{O}}\left(l_{r}^{\prime} \underline{\mathrm{O}}\left(l_{r}^{\prime} \underline{\mathrm{O}} l_{r}\right)\right)\right)\right) \underline{\mathrm{O}} l_{r}\right)} \mathcal{F}_{\mathbf{H}}(\vartheta) \\
& =\bigcap_{\vartheta \in\left(\left(l_{r} \underline{\mathrm{O}}\left(((y \underline{\mathrm{O}} e) \underline{\mathrm{O}} x) \underline{\mathrm{O}}\left(l_{r}^{\prime} \underline{\mathrm{O}}\left(l_{r}^{\prime} \underline{\mathrm{O}} l_{r}\right)\right)\right)\right) \underline{\mathrm{O}} l_{r}\right)} \mathcal{F}_{\mathbf{H}}(\vartheta) \\
& \supseteq \mathcal{F}_{\mathbf{H}}\left(l_{r}\right) \cap \mathcal{F}_{\mathbf{H}}\left(l_{r}\right) \\
& =\mathcal{F}_{\mathbf{H}}\left(l_{r}\right) \text {. }
\end{aligned}
$$

Therefore, $\mathcal{F}_{\mathbf{H}}$ is an S.I. interior-hyperideal of $\mathbf{H}$ over $\mathcal{U}$.

Conclusion. In this paper, we have introduced soft interior-hyperideals in LAsemihypergroups and characterized left regular LA-semihypergroups in terms of soft interior-hyperideals. Based on the results of this paper, some further work can be done on the properties of soft interior-hyperideals in other structures.

\section{REFERENCES}

[1] H. Aktas and N. Cagman, Soft sets and soft groups, Inform. Sci. 177 (2007), 2726-2735.

[2] V. Amjad, K. Hila and F. Yousafzai, Generalized hyperideals in locally associative left almost semihypergroups, New York J. Math. 20 (2014), 1063-1076.

[3] V. Amjad and F. Yousafzai, On pure LA-semihypergroups, Konuralp J. Math. 2 (2014), 53-63.

[4] S. M. Anvariyeh, S. Mirvakili, O. Kazanci and B. Davvaz, Algebraic hyperstructures of soft sets associated to semihypergroups, Southeast Asian Bull. Math. 35 (2011), 911-925. 
[5] M. Aslam, T. Aroob and N. Yaqoob, On cubic G-hyperideals in left almost G-semihypergroups, Ann. Fuzzy Math. Inform. 5 (2013), 169-182.

[6] M. Azhar, M. Gulistan, N. Yaqoob and S. Kadry, On fuzzy ordered LA-semihypergroups, International Journal of Analysis and Applications 16 (2018), 276-289.

[7] N. Cagman, F. Citak and H. Aktas, Soft int-group and its applications to group theory, Neural Computing and Applications 21 (2012), 151-158.

[8] N. Cagman and S. Enginoglu, Soft set theory and uni-int decision making, European J. Oper. Res. 207 (2010), 848-855.

[9] P. Corsini, Prolegomena of Hypergroup Theory, Second Edition, Aviani Editore, Aviani, 1993.

[10] B. Davvaz and V. L. Fotea, Hyperring Theory and Applications, International Academic Press, Gulf Breeze, Fla, USA, 2007.

[11] M. Farooq, A. Khan, and B. Davvaz, Characterizations of ordered semihypergroups by the properties of their intersectional-soft generalized bi-hyperideals, Soft Computing 9(22) (2018), 3001-3010.

[12] F. Feng, Y. B. Jun and X. Zhao, Soft semirings, Comput. Math. Appl. 56 (2008), 2621-2628.

[13] M. Gulistan, M. Khan, N. Yaqoob and M. Shahzad, Structural properties of cubic sets in regular LA-semihypergroups, Fuzzy Inf. Eng. 9 (2017), 93-116.

[14] M. Gulistan, N. Yaqoob and M. Shahzad, A note on Hv-LA-semigroups, Politehn. Univ. Bucharest Sci. Bull. Ser. A Appl. Math. Phys. 77 (2015), 93-106.

[15] A. Hasankhani, Ideals in a semihypergroup and greens relations, Ratio Mathematica 13 (1999), 29-36.

[16] K. Hila and J. Dine, On hyperideals in left almost semihypergroups, ISRN Algebra 2011 (2011), Article ID 953124, 8 pages.

[17] M. A. Kazim and M. Naseeruddin, On almost semigroups, Aligarh Bull. Math. 2 (1972), 1-7.

[18] A. Khan, M. Farooq, M. Izhar and B. Davvaz, Fuzzy hyperideals of left almost semihypergroups, International Journal of Analysis and Applications 15 (2016), 155-171.

[19] M. Khan, N. Y. M. Gulistan and F. Hussain, General cubic hyperideals of LA-semihypergroups, Afr. Mat. 27 (2016), 731-751.

[20] M. Khan, N. Y. M. Gulistan and F. Hussain, General cubic hyperideals of LA-semihypergroups, Afr. Mat. 27 (2016), 731-751.

[21] S. A. Khan, M. Y. Abbasi and A. F. Talee, A new approach to soft hyperideals in LAsemihypergroups, in: N. Yadav, A. Yadav, J. C. Bansal, K. Deep and J. H. Kim (Eds.), Harmony Search and Nature Inspired Optimization Algorithm, Theory and Applications, ICHSA-2018, Advances in Intelligent System and Computing 29 (2019), 293-303, DOI 10.1007/978-981-130761-4-29.

[22] P. K. Maji, R. Biswas and A. R. Roy, Soft set theory, Comput. Math. Appl. 45 (2003), 555-562.

[23] F. Marty, Sur une generalization de la notion de group, in: Proceedings of 8th Congress Math. Scandinaves, Stockholm, Sweden, 1934, 45-49.

[24] D. Molodtsov, Soft set theory-first results, Comput. Math. Appl. 37 (1999), 19-31.

[25] S. Naz and M. Shabir, On soft semihypergroups, Journal of Intelligent and Fuzzy Systems 26 (2014), 2203-2213.

[26] A. Sezgin, A new approach to LA-semigroup theory via the soft sets, Journal of Intelligent and Fuzzy Systems 26 (2014), 2483-2495.

[27] A. Sezgin and A. O. Atagun, On operations of soft sets, Comput. Math. Appl. 61 (2011), $1457-1467$.

[28] N. Yaqoob, M. Akram and M. Aslam, Intuitionistic fuzzy soft groups induced by $(t, s)$-norm, Indian Journal of Science and Technology 6 (2013), 4282-4289.

[29] N. Yaqoob, M. Aslam and Faisal, On soft G-hyperideals over left almost G-semihypergroups, Journal of Advanced Research in Dynamical and Control Systems 4 (2012), 1-12. 
[30] N. Yaqoob, P. Corsini and F. Yousafzai, On intra-regular left almost semihypergroups with pure left identity, J. Math. 2013 (2013), Article ID 510790, 10 pages.

[31] N. Yaqoob and M. Gulistan, Partially ordered left almost semihypergroups, J. Egyptian Math. Soc. 23 (2015), 231-235.

[32] F. Yousafzai, K. Hila, P. Corsini and A. Zeb, Existence of non-associative algebraic hyperstructures and related problems, Afr. Mat. 26 (2015), 981-995.

[33] F. Yousafzai and C. Piersiulio, Some characterization problems in LA-semihypergroups, Journal of Algebra, Number Theory: Advances and Applications 10 (2013), 41-55.

[34] F. Yousafzai, N. Yaqoob and A. Ghareeb, Left regular AG-groupoids in terms of fuzzy interior ideals, Afr. Mat. 24 (2013), 577-587.

[35] L. A. Zadeh, Fuzzy sets, Information and Control 8 (1965), 338-353.

${ }^{1}$ Department of MATHEMATiCs,

Jamia Millia Islamia,

NeW DELHI-110 025, IndiA

Email address: yahya_alig@yahoo.co.in

Email address: khansabahat361@gmail.com

Email address: fuzzyaakif786.jmi@gmail.com

${ }^{2}$ Department of Mathematics, Abdul Wali Khan University, MARDAn, KP, PAKISTAN

Email address: azhar4set@yahoo.com 